\title{
Anomalous propulsion regime in axonemal-propelled cargoes
}

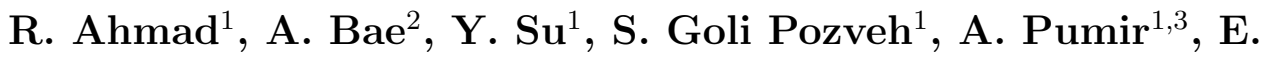 \\ Bodenschatz $^{1,4,5}$, and A. Gholami ${ }^{1 *}$ \\ ${ }^{1}$ Max Planck Institute for Dynamics and Self-Organization, Am Fassberg 17, \\ D-37077 Göttingen, Germany \\ ${ }^{2}$ Department of Biomedical Engineering, University of Rochester, USA \\ ${ }^{3}$ Laboratoire de Physique, Ecole Normale Suprieure de Lyon, Universit Lyon 1 and \\ CNRS, F-69007 Lyon, France \\ ${ }^{4}$ Institute for Nonlinear Dynamics, University of Göttingen, D-37073 Göttingen, \\ Germany \\ ${ }^{5}$ Laboratory of Atomic and Solid-State Physics and Sibley School of Mechanical and \\ Aerospace Engineering, Cornell University, Ithaca, New York 14853, USA
}

E-mail: azam.gholami@ds.mpg.de

May 2021

\begin{abstract}
Bio-actuated micro-swimmers provide a platform to understand physical principles related to the motion of micro-organisms at low Reynolds numbers. Here, we used isolated and demembranated flagella from green algae Chlamydomonas reinhardtii as an ATP-fueled bio-actuator for propulsion of micron-sized beads. Chlamydomonas flagella have an asymmetric waveform, which can be accurately described as a superposition of a static component corresponding to an arc-shaped intrinsic curvature, a mode describing the global oscillations of the axonemal curvature, and a main baseto-tip traveling wave component. By decomposing experimental beat patterns in Fourier modes, and applying resistive force theory, we performed numerical simulations and obtained analytical approximations for the mean rotational and translational velocities of a flagellum-propelled bead. Our analysis reveals the existence of a counterintuitive anomalous propulsion regime where the speed of the flagellum-driven cargo increases with increasing the cargo size. Further, it demonstrates that in addition to the intrinsic curvature and even harmonics, asymmetric bead-flagellum attachment also contributes in the rotational velocity of the micro-swimmer. This turning mechanism induced by sideways cargo attachment has potential applications in fabrication of bioactuated medical micro-robots in the subject of targeted drug delivery and synthetic micro-swimmers.
\end{abstract}

Keywords: Chlamydomonas reinhardtii, Flagella wave dynamics, Mode analysis, Bioactuated micro-swimmers, Anomalous propulsion 


\section{Introduction}

Ancient organelles cilia and flagella are important eye-lashed shaped cellular protrusions which perform periodic whip-like motion to generate fluid flows and motility [1,2]. In many organisms, multiple motile cilia coordinate their beating activity to enhance the swimming efficiency or directional fluid transport. Examples are transport of cerebrospinal fluid, which contains physiologically important signaling molecules, through the ventricular cavities of the mammalian's brain [3], mucus clearance in mammalian airways [4], or the bi-flagellated alga Chlamydomonas reinhardtii ( $C$. reinhardtii) which swims in water with its two flagella using a characteristic breaststroke beat pattern $[5-9]$.

Cilia and flagella have a tube-shaped structure consisting of a periodic arrangement of nine microtubule doublets (MTDs) at the periphery and two microtubule singlets at the center. This $9+2$ structure, known as axoneme, has the diameter of $200 \mathrm{~nm}$ and can be actuated via coordinated collective activity of dynein molecular motors that in one beat cycle, convert chemical energy of around $10^{5}$ ATP molecules into mechanical work by sliding neighboring MTDs [10-13]. However, the sliding of MTDs is resisted by structural constraints imposed by nexin linker proteins which mechanically couple outer MTDs, and protein complexes "radial spokes" which extend toward the central pair [14-18]. Instead, sliding converts to rhythmic bending deformations that propagate along the axonemal length.

In nature, flagella in different species show variety of beat patterns. In contrast to spermatozoa, flagella isolated from green algae $C$. reinhardtii beat by asymmetric bending deformations propagating at the fundamental beat frequency along the axonemal length in a base-to-tip direction [19-23]. Here, 'asymmetric waveform' means that the flagellar shapes averaged over one beat cycle results to a semicircular arc with mean curvature of around $-0.19 \mu \mathrm{m}^{-1}$, leading to a curved swimming trajectory $[21,24,25]$. In the absence of this static component, the flagella swims in a straight sperm-like trajectory [19,20]. In our recent study [26], we have shown that in addition to the static curvature, the base-to-tip traveling wave component and its higher harmonics, two other modes also play a crucial role. First, a time-symmetric mode describing the global oscillations of the axonemal curvature. Second, a tip-to-base wave component that propagates opposite to the main traveling mode at the fundamental beat frequency, albeit with a reduced amplitude. The time-symmetric mode by itself can not contribute to the propulsion (scallop theorem [27]), but once the time symmetry is broken by other traveling wave components, it enhances the translational and rotational velocities of the axoneme with a factor of around $2[26]$.

In this study, we demonstrate the feasibility of using isolated and demembranated flagella from C.reinhardtii as a bio-actuator for propulsion of micron-sized beads. The swimming trajectory of the flagellum-driven bead depends on the flagellar waveform. Here, we combine experiments, numerical simulations and analytical approximations to investigate the effect of (i) various wave components, (ii) the bead size, and (iii) 
symmetric versus asymmetric bead-axoneme attachment on the swimming dynamics of a bead propelled by an ATP-reactivated axoneme. By describing the axonemal waveform as a combination of a static curvature, a main base-to-tip traveling wave component, and a time-symmetric mode highlighting the global oscillations of the axonemal curvature, we simulate the swimming trajectory of an axonemal-propelled bead using resistiveforce theory $[28,29]$ and obtain analytical approximations for the translational and rotational velocities of the swimmer in the limit of small amplitude of curvature waves. Our analysis reveals a surprising non-monotonic behavior of the mean translational and rotational velocities of the axonemal-driven bead as a function of the bead radius. It also shows that for a freely swimming axoneme which rotates predominantly with its static mode of the axonemal waveform, sideways bead attachment is sufficient to generate mean rotational velocities comparable to the rotation rates induced by the intrinsic curvature. Finally, we used calcium ions to reduce the static curvature of axonemes $[19,20]$, inducing a transition from circular to straight sperm-like swimming trajectories.

\section{Materials and Methods}

\subsection{Isolation of axonemes from $C$. reinhardtii and attachment to the beads}

Isolation and reactivation of axonemes are done based on the standard protocols in Refs. [30,31]. Briefly, wild-type C. reinhardtii cells, strain SAG 11-32b, are grown axenically in TAP (tris-acetate-phosphate) medium on a $12 \mathrm{~h} / 12 \mathrm{~h}$ day-night cycle. Upon adding Dibucaine, cells release their flagella which we purify on a $25 \%$ sucrose cushion, and demembranate them using detergent NP-40 in HMDEK solution (30 mM HEPES-KOH, 5 mM MgSO 4 , 1 mM DTT, 1 mM EGTA, 50 mM potassium acetate, $\mathrm{pH}$ 7.4) supplemented with $0.2 \mathrm{mM}$ Pefabloc. The membrane-free axonemes are resuspended in HMDEK buffer plus $1 \%(\mathrm{w} / \mathrm{v})$ polyethylene glycol $\left(\mathrm{M}_{w}=20 \mathrm{~kg} \mathrm{~mol}^{-1}\right), 0.2 \mathrm{mM}$ Pefabloc and use freshly after isolation. To perform reactivation experiments, we dilute axonemes in HMDEKP reactivation buffer (HMDEK plus $1 \%$ PEG) supplemented with $200 \mu \mathrm{M}$ ATP. For bead attachment, beads are incubated for $20 \mathrm{~min}$ with axonemes before being mixed with reactivation buffer and infused into $100 \mu \mathrm{m}$ deep flow chambers, built from cleaned glass and double-sided tape. Glass slides are treated with casein $(2 \mathrm{mg} / \mathrm{mL}$

in HMDEKP buffer) for 5 min before use to avoid axoneme-substrate attachment. For calcium experiments, we add $0.1 \mathrm{mM} \mathrm{CaCl}_{2}$ to the reactivation buffer HMDEKP.

\subsection{Axoneme Tracking}

We used high-speed phase-contrast microscopy to capture high-resolution images of fast beating axonemes (100X objective, $1000 \mathrm{fps}$ ). In these images, the bead appears as a bright spot, which interferes with the tracking algorithm of the axoneme (Fig. S5). Therefore, we first threshold the images to remove the bead. Next, to increase the signal to noise ratio, we invert phase-contrast images and subtract the mean-intensity 
of the time series [23]. To smooth the images, we apply a Gaussian filter. Tracking of axonemes are done using the gradient vector flow (GVF) technique [32,33]. For the first frame, we select a region of interest that includes only one actively beating axoneme. Then, to initialize the snake, we draw a line polygon along the contour of the axoneme in the first frame (see supplemental Fig. S5). This polygon is interpolated at $N$ equally spaced points and used as a starting parameter for the snake. The GVF is calculated using the GVF regularization coefficient $\mu=0.1$ with 20 iterations. The snake is then deformed according to the GVF where we have adapted the original algorithm by Xu and Prince for open boundary conditions $[32,33]$. The axoneme is characterized by $N$ points along the axonemal length $s$ so that $s=0$ is the basal region and $s=L$ is the distal tip. Here $L$ is the total contour length of the filament. The position of axoneme at contour length $s_{i}$ is given by $r\left(s_{i}\right)=\left(x\left(s_{i}\right), y\left(s_{i}\right)\right)$.

\subsection{Resistive force theory and calculation of mean translational and rotational velocities}

The fluid motion involved during the swimming of small objects is characterized by very small Reynolds numbers, so the viscous forces and torques are much larger than the inertial forces $[27,34]$. Newton's laws then consist of an instantaneous balance between external and fluid forces and torques exerted on the swimmer, i.e. $\mathcal{F}_{\text {ext }}+\mathcal{F}_{\text {fluid }}=0$ and $\mathcal{T}_{\text {ext }}+\mathcal{T}_{\text {fluid }}=0$. The micro-swimmer in our system is formed of an axoneme (a filament of length $L \sim 10 \mu \mathrm{m}$ and radius $100 \mathrm{~nm}$ ), which is attached from one end to a micron-sized bead. The force $\mathcal{F}_{\text {fluid }}$ and torque $\mathcal{T}_{\text {fluid }}$ exerted by the fluid on the axoneme-bead swimmer can be written as:

$$
\begin{aligned}
& \mathcal{F}_{\text {fluid }}=\mathcal{F}_{\text {Bead }}+\int_{0}^{L} d s \mathcal{F}_{\text {Axoneme }}(s, t), \\
& \mathcal{T}_{\text {fluid }}=\mathcal{T}_{\text {Bead }}+\int_{0}^{L} d s \mathbf{r}(s, t) \times \mathcal{F}_{\text {Axoneme }}(s, t),
\end{aligned}
$$

where $\mathcal{F}_{\text {bead }}$ and $\mathcal{T}_{\text {Bead }}$ are the hydrodynamic drag force and torque acting on the bead and the integrals over the contour length $L$ of the axoneme calculate the total hydrodynamic force and torque exerted by the fluid on the axoneme. The bead is propelled by oscillatory shape deformation of the ATP-reactivated axoneme. At any given time, we consider axoneme-bead swimmer as a solid body with unknown translational and rotational velocities $\mathbf{U}(t)$ and $\Omega(t)$ yet to be determined. $\mathcal{F}_{\text {fluid }}$ and $\mathcal{T}_{\text {fluid }}$ can be separated into propulsive part due to relative shape deformations of the axoneme in body-fixed frame and drag part [35]:

$$
\left(\begin{array}{c}
\mathcal{F}_{\text {fluid }} \\
\mathcal{T}_{\text {fluid }}
\end{array}\right)=\left(\begin{array}{c}
\mathcal{F}^{\text {prop }} \\
\mathcal{T}^{\text {prop }}
\end{array}\right)-\mathcal{D}\left(\begin{array}{l}
\mathbf{U} \\
\Omega
\end{array}\right)=\left(\begin{array}{c}
\mathcal{F}^{\text {prop }} \\
\mathcal{T}^{\text {prop }}
\end{array}\right)-\left(\mathcal{D}_{A}+\mathcal{D}_{B}\right)\left(\begin{array}{l}
\mathbf{U} \\
\Omega
\end{array}\right)
$$

where $6 \times 6$ geometry-dependent drag matrix $\mathcal{D}$ is symmetric and nonsingular (invertible) and is composed of drag matrix of the axoneme $\mathcal{D}_{A}$ and drag matrix of the bead $\mathcal{D}_{B}$. 
We also note that a freely swimming axoneme-bead experiences no external forces and torques, thus $\mathcal{F}_{\text {fluid }}$ and $\mathcal{T}_{\text {fluid }}$ must vanish. Further, since swimming effectively occurs in $2 \mathrm{D}, \mathcal{D}$ is reduced to a $3 \times 3$ matrix and Eq. 3 can be reformulated as:

$$
\left(\begin{array}{c}
\mathbf{U}_{x} \\
\mathbf{U}_{y} \\
\Omega_{z}
\end{array}\right)=\left(\mathcal{D}_{A}+\mathcal{D}_{B}\right)^{-1}\left(\begin{array}{c}
\mathcal{F}_{x}^{\text {prop }} \\
\mathcal{F}_{y}^{\text {prop }} \\
\mathcal{T}_{z}^{\text {prop }}
\end{array}\right)
$$

which we use to calculate translational and rotational velocities of the swimmer after determining the drag matrices $\mathcal{D}_{A}$ and $\mathcal{D}_{B}$, and the propulsive forces and torque $\left(\mathcal{F}_{x}^{\text {prop }}, \mathcal{F}_{y}^{\text {prop }}, \mathcal{T}_{z}^{\text {prop }}\right)$.

We calculate $\mathcal{F}_{x}^{\text {prop }}, \mathcal{F}_{y}^{\text {prop }}$ and $\mathcal{T}_{z}^{\text {prop }}$ in the body-fixed frame by selecting the basal end of the axoneme (bead-axoneme contact point) as the origin of the swimmer-fixed frame. As shown in Figs. 1A and 2A, we define the local tangent vector at contour length $s=0$ as $\hat{X}$ direction, the local normal vector $\hat{n}$ as $\hat{Y}$ direction, and assume that $\hat{z}$ and $\hat{Z}$ are parallel. We define $\theta_{0}(t)=\theta(s=0, t)$ as the angle between $\hat{x}$ and $\hat{X}$ which gives the velocity of the bead in the laboratory frame as $\mathbf{U}_{x}^{\text {Bead }}=\cos \theta_{0}(t) \mathbf{U}_{x}+\sin \theta_{0}(t) \mathbf{U}_{y}$ and $\mathbf{U}_{y}^{\text {Bead }}=-\sin \theta_{0}(t) \mathbf{U}_{x}+\cos \theta_{0}(t) \mathbf{U}_{y}$. Furthermore, note that the instantaneous velocity of the axoneme in the lab frame is given by $\mathbf{u}=\mathbf{U}+\Omega \times \mathbf{r}(s, t)+\mathbf{u}^{\prime}$, where $\mathbf{u}^{\prime}$ is the deformation velocity of flagella in the body-fixed frame, $U=\left(\mathbf{U}_{x}, \mathbf{U}_{y}, 0\right)$ and $\Omega=\left(0,0, \Omega_{z}\right)$ with $\Omega_{z}=d \theta_{0}(t) / d t$.

To calculate $\mathcal{F}_{x}^{\text {prop }}, \mathcal{F}_{y}^{\text {prop }}$ and $\mathcal{T}_{z}^{\text {prop }}$ for a given beating pattern of axoneme in the body-fixed frame, we used the classical framework of resistive force theory (RFT), which neglects long-range hydrodynamic interactions between different parts of the flagellum as well as the inter-flagella interactions $[28,29]$. In this theory, each flagellum is divided to small cylindrical segments moving with velocity $\mathbf{u}^{\prime}(s, t)$ in the body-frame and the propulsive force $\mathcal{F}^{\text {prop }}$ is proportional to the local center-line velocity components of each segment in parallel and perpendicular directions:

$$
\begin{aligned}
\mathcal{F}^{\mathrm{prop}}(s, t) & =\zeta_{\|} \mathbf{u}_{\|}^{\prime}(s, t)+\zeta_{\perp} \mathbf{u}_{\perp}^{\prime}(s, t), \\
\mathbf{u}_{\|}^{\prime}(s, t) & =[\dot{\mathbf{r}}(s, t) \cdot \mathbf{t}(s, t)] \mathbf{t}(s, t), \\
\mathbf{u}_{\perp}^{\prime}(s, t) & =\dot{\mathbf{r}}(s, t)-\mathbf{u}_{\|}^{\prime}(s, t),
\end{aligned}
$$

where $\mathbf{u}_{\|}^{\prime}$ and $\mathbf{u}_{\perp}^{\prime}$ are the projection of the local velocity on the directions parallel and perpendicular to the axoneme. The friction coefficients in parallel and perpendicular directions are defined as $\zeta_{\|}=4 \pi \mu /(\ln (2 L / a)+0.5)$ and $\zeta_{\perp}=2 \zeta_{\|}$, respectively. For a water-like environment with viscosity $\mu=0.96 \mathrm{pN} \mathrm{msec} / \mu \mathrm{m}^{2}$, we obtain $\zeta_{\|} \sim 2.1 \mathrm{pN} \mathrm{msec} / \mu \mathrm{m}^{2}$.

\subsection{Drag matrix of a bead in 2D}

Let us consider the two-dimensional geometry defined in Fig. 1A. Note that the origin of the swimmer-fixed frame is not at the bead center and is selected to be at the beadaxoneme contact point, as shown in Fig. 1A. In general, the tangent vector at position 


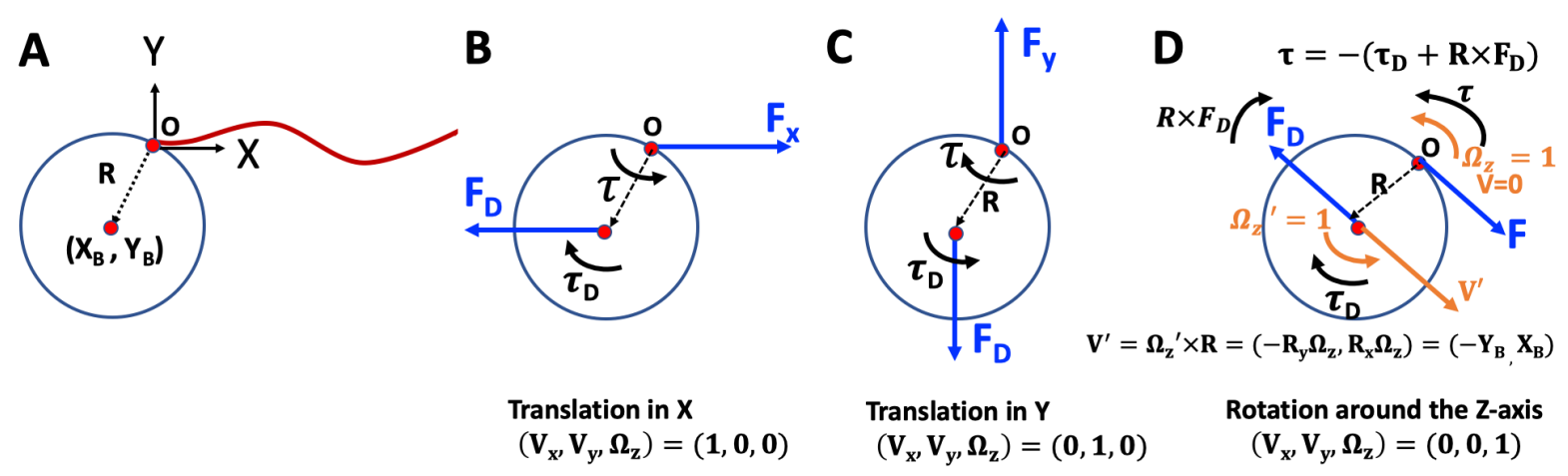

Figure 1. A) Illustration of the bead orientation with respect to the axoneme and definition of the swimmer-fixed frame. Tangent vector at $s=0$ (basal end) defines the $X$ direction. B-D) Schematic drawing of the forces and torques that counteract the hydrodynamic drag force and torque.

$s=0$ of the axoneme, which defines the $X$-axis, does not pass through the bead center located at $\left(X_{B}, Y_{B}\right)$. This asymmetric bead-axoneme attachment is also observed in our experiments, as shown in Fig. 2A. We emphasize that the drag force is actually a distributed force, given by $d f=\sigma \cdot d \vec{A}$ applied at the surface of the sphere, but symmetry implies that drag force effectively acts on the bead center. We define the translational and rotational friction coefficients of the bead as $\nu_{T}=6 \pi \alpha_{t} \mu R$ and $\nu_{R}=8 \pi \alpha_{r} \mu R^{3}$, where factors $\alpha_{t}=1 /\left(1-9(R / h) / 16+(R / h)^{3} / 8\right)$ and $\alpha_{r}=1 /\left(1-(R / h)^{3} / 8\right)$ are corrections due to the fact that axonemal-based bead propulsion occurs in the vicinity of a substrate [36]. Here $R$ is the bead radius $(\sim 0.5 \mu m), h$ is the distance between the center of the bead and the substrate. Assuming $R / h \sim 1$, we obtain $\alpha_{t}=16 / 9$ and $\alpha_{r}=8 / 7$. We now look at each component of velocity and ask what force do we need to apply to counteract the drag force?

(i) Translation in $\mathrm{X}$-direction. In this case, we have $\left(V_{x}, V_{y}, \Omega_{z}\right)=(1,0,0)$ as shown in Fig. 1B. We need to apply a force in $+\mathrm{X}$ direction to counteract the drag force as:

$$
F_{x}=6 \pi \alpha_{t} \mu R=\nu_{T}
$$

But we must also apply a torque in $+\mathrm{Z}$ direction for the case illustrated in Fig. 1B (where $Y_{B}<0$ ) to prevent rotation from occurring:

$$
\tau=-\tau_{D}=-Y_{B} F_{x}=-\nu_{T} Y_{B}
$$

so we get:

$$
\left(F_{x}, F_{y}, \tau\right)=\left(\nu_{T}, 0,-\nu_{T} Y_{B}\right) V_{x}
$$

(ii) Translation in Y-direction. This case corresponds to $\left(V_{x}, V_{y}, \Omega_{z}\right)=(0,1,0)$ as shown in Fig. 1C. We have $F_{x}=0$ and $F_{y}=+6 \pi \alpha_{t} \mu R=\nu_{T}$. Note that we need to apply a negative torque, and since $X_{B}<0$, we have $\tau=+X_{B} \nu_{T}$ which gives:

$$
\left(F_{x}, F_{y}, \tau\right)=\left(0, \nu_{T}, \nu_{T} X_{B}\right) V_{y}
$$


(iii) Rotation around Z-direction. For this case, we have $\left(V_{x}, V_{y}, \Omega_{z}\right)=(0,0,1)$ as shown in Fig. 1D. Before looking at the forces, let us examine the motion. The rotation $\Omega^{\prime}=1$ of the bead center around the origin $O$ also generates translational velocity $V^{\prime}=\left(-R_{y}, R_{x}\right) \Omega=\left(-Y_{B}, X_{B}\right)$; see Fig. 1D. Note that for $Y_{B}<0$ and $X_{B}<0$, we get $V_{x}^{\prime}>0$ and $V_{y}^{\prime}<0$ which is consistent. Around the center of the bead, drag exerts force and torque $F_{D}$ and $\tau_{D}$, as depicted in Fig. 1D:

$$
\overrightarrow{F_{D}}=-v_{T} \overrightarrow{V^{\prime}}=\left(\nu_{T} Y_{B},-\nu_{T} X_{B}\right), \quad \text { and } \quad\left(\vec{\tau}_{D}\right)_{Z}=-\nu_{R}
$$

To counteract the drag force, we must apply:

$$
\overrightarrow{F_{D}}=\left(-\nu_{T} Y_{B}, \nu_{T} X_{B}\right), \quad \text { and } \quad \tau_{Z}=\nu_{R}+F R=\nu_{R}+\nu_{T} R^{2}
$$

so we obtain $\left(F_{x}, F_{y}, \tau_{z}\right)=\left(-\nu_{T} Y_{B}, \nu_{T} X_{B}, \nu_{R}+\nu_{T} R^{2}\right) \Omega_{z}$.

This can be expressed as:

$$
\left(\begin{array}{c}
F_{x} \\
F_{y} \\
\tau_{z}
\end{array}\right)=\left(\begin{array}{ccc}
\nu_{T} & 0 & -\nu_{T} Y_{B} \\
0 & \nu_{T} & \nu_{T} X_{B} \\
-\nu_{T} Y_{B} & \nu_{T} X_{B} & \nu_{R}+\nu_{T} R^{2}
\end{array}\right)\left(\begin{array}{c}
V_{x} \\
V_{y} \\
\Omega_{z}
\end{array}\right)
$$

For the special case that center of the bead is at $\left(X_{B}, Y_{B}\right)=(-R, 0)$ which corresponds to the situation that tangent vector of the flagella at $s=0$ goes through the bead center, Eq. 11 simplifies to:

$$
\left(\begin{array}{c}
F_{x} \\
F_{y} \\
\tau_{z}
\end{array}\right)=\left(\begin{array}{ccc}
\nu_{T} & 0 & 0 \\
0 & \nu_{T} & -\nu_{T} R \\
0 & -\nu_{T} R & \nu_{R}+\nu_{T} R^{2}
\end{array}\right)\left(\begin{array}{c}
V_{x} \\
V_{y} \\
\Omega_{z}
\end{array}\right)
$$

Note that Eqs. 11-12 present the forces and torque exerted by the bead on the fluid which has opposite sign of the forces generated by the fluid on the bead, so the drag matrix of the bead $\mathcal{D}_{B}$ is given by:

$$
\mathcal{D}_{B}=\left(\begin{array}{ccc}
-\nu_{T} & 0 & \nu_{T} Y_{B} \\
0 & -\nu_{T} & -\nu_{T} X_{B} \\
\nu_{T} Y_{B} & -\nu_{T} X_{B} & -\nu_{R}-\nu_{T} R^{2}
\end{array}\right) .
$$

The general form of the drag matrix in $3 \mathrm{D}$ is derived in supplemental information.

\section{Results}

\subsection{An axoneme-propelled bead}

We fabricated $C$. reinhardtii flagella-based propelled beads by incubating isolated and demembranated axonemes ( $10 \mu \mathrm{m}$ in length) with beads of diameter $1 \mu \mathrm{m}$ (see Materials and Methods). In most cases, a single bead attaches to the basal or the distal tips of axonemes, but in very rare cases, we also observed that more than one bead 

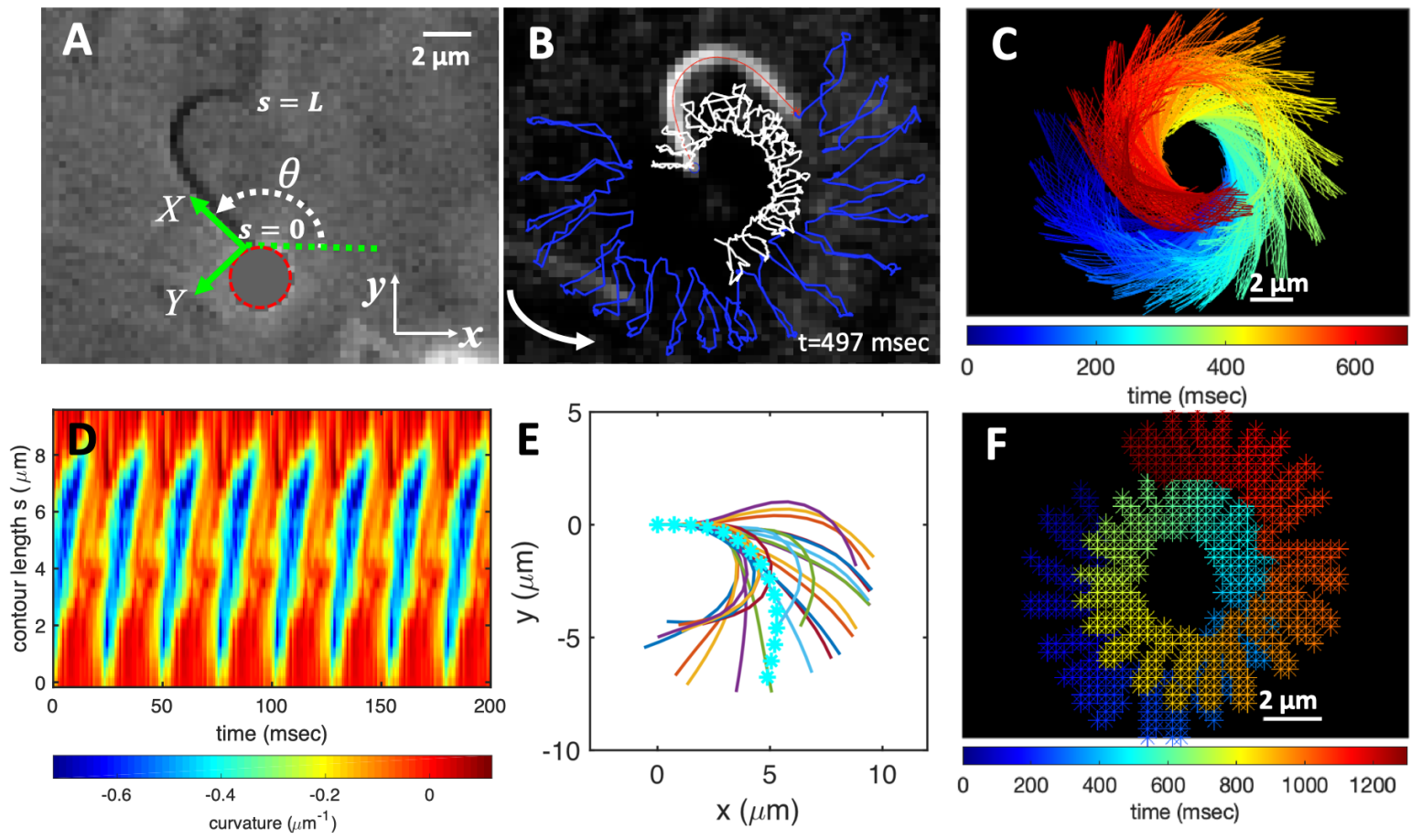

Figure 2. A) An exemplary axoneme attached at the basal end $(s=0)$ to a bead of diameter $1 \mu \mathrm{m}$. B) Tracked axoneme and trajectories of its distal (blue lines) and basal (white lines) ends. Axoneme-bead swimmer rotates counter-clockwise while the bead center follows a helical path. C) Color-coded time projection of the axonemal shapes in panel B showing a circular swimming path. D) Curvature waves as they travel at the frequency of $38.24 \mathrm{~Hz}$ from the proximal region toward the distal tip. E) Mean shape of the axoneme in cyan color averaged over one beat cycle showing a circular arc with static curvature of $\sim-0.19 \mu \mathrm{m}^{-1}$. At different time points, flagella is translated and rotated such that the basal end is at $(0,0)$ and the tangent vector at $s=0$, which defines the $X$-axis in the swimmer-fixed frame, is along the $x$-axis of the laboratory-fixed frame (see panel A). F) Color-coded trajectory of the bead center showing a helical trajectory. Global rotational frequency of the swimmer is $\sim 1.6 \mathrm{~Hz}$ $(\sim 1$ full rotation in around $640 \mathrm{msec}$; see also Videos 1,2).

attach either to the tips or along the axonemal length. In most cases, the attachment of the beads to the basal region of the axoneme was asymmetric, i.e. the tangent vector of the axoneme at the bead-axoneme contact point does not pass through the bead center (Fig. 2A and Videos 1-2). The consequences of this asymmetric versus symmetric attachment are discussed in the next section.

The swimming dynamics of an axonemal-propelled bead differs from sperm flagellar propulsion primarily in that isolated axonemes from $C$. reinhardtii are smaller in length $(10 \mu \mathrm{m}$ versus $50 \mu \mathrm{m})$ and they beat faster with a non-zero static curvature of $\kappa_{0}$ around $-0.19 \mu \mathrm{m}^{-1}$ (Fig. 2E), causing a circular swimming trajectory (see Fig. 2B-C). The negative sign of $\kappa_{0}$ indicate a clockwise bend when moving from the basal end at $s=0$ toward the distal tip at $s=L$ (Fig. 2E). The base-to-tip bending deformations superimposed on this negative intrinsic curvature cause a counter-clockwise rotation of 
the axoneme (Fig. 2B). Beating frequency of axonemes depends on ATP concentration following a Michaelis-Menten-type kinetics [23,37] with a linear trend at low amount of ATP and saturation at higher ATP concentrations around $1 \mathrm{mM}$. Following the addition of ATP, asymmetric bending waves initiate at the basal end (which is attached to a bead in Fig. 2) and travel toward the distal tip at frequency of $f_{0} \sim 38.24 \mathrm{~Hz}$ (Fig. 2D). The traveling periodic curvature waves provide the necessary thrust to propel the bead in the surrounding water-like fluid. This thrust force is balanced with the viscous drag exerted by the fluid on the swimmer, as discussed in the Sec. 2.3. The flagellar beat is effectively in 2D and is characterized by a circular swimming trajectory and translation in the laboratory frame of reference, and the combination of rotation and translation results in a helical swimming path of the bead. This helical trajectory of the bead is shown in Fig. 2F. Note that the $x-y$ positions of the bead center in Fig. $2 \mathrm{~F}$ exhibit tiny oscillations at the fast beat frequency of $f_{0} \sim 38.24 \mathrm{~Hz}$ and a secondary slow global rotational frequency around $1.6 \mathrm{~Hz}$.

We define the dimensionless curvature as $C(s, t)=\kappa(s, t) L /(2 \pi)$, where $\kappa(s, t)$ is the curvature at arc-length $0 \leq s \leq L$ along the axonemal length at time-point $t$. Fig. 3A shows the power spectrum in time (horizontal) and space (vertical) of the "windowed" curvature waves of the representative axoneme, shown in Fig. 2. The windowing procedure used here consists in repeating a given beat cycle of the axoneme an integer number of times [26]. The corresponding 2D power spectrum shows several dominant peaks. The most dominant one is at the zero spatial and temporal frequencies which highlights the intrinsic curvature of the axoneme $\kappa_{0}$. Note that with $\kappa_{0} \sim 0.19 \mu \mathrm{m}^{-1}$ and axonemal length of $L \sim 9.4 \mu \mathrm{m}$, we expect $C_{0}=\kappa_{0} L /(2 \pi)$ to have the magnitude of around 0.28 . The second dominant peak occurs at the fundamental beat frequency $f_{0}$ with the spatial frequency/wavelength of $L$. Interestingly, we also observe a peak at zero spatial frequency (infinite wavelength) at beat frequency $f_{0}$ [26]. This mode $C_{0}^{\prime}$ describes the global oscillations of the axonemal curvature and has a higher amplitude than the second harmonic $C_{2}$ and the back-propagating wave component $C_{1}^{\prime}$ (see Fig. 3B). We also note that $C_{1}^{\prime}$ and $C_{2}$ modes have both comparable, but small amplitudes and the shape dynamics is dominated by $C_{0}, C_{0}^{\prime}$ and the main base-to-tip traveling wave component $C_{1}$. These three modes provide a qualitatively satisfactory description of the axonemal shapes (Fig. 3G). For comparison, reconstructed shapes with all the five modes $C_{0}$, $C_{0}^{\prime}, C_{1}, C_{1}^{\prime}$ and $C_{2}$ (Fig. 3E) are also presented. Please note that the $C_{0}^{\prime}$ mode, which describes the global oscillations of the axonemal curvature, has a significant contribution in reconstructing the axonemal shapes and in contrast to $C_{1}^{\prime}$ and $C_{2}$ modes, removing $C_{0}^{\prime}$ mode shows a strong qualitative effect on the reconstructed shapes (Fig. $3 \mathrm{H}$ ).

\subsection{Analytical approximations of rotational and translational velocities of an axonemal-propelled bead}

To understand the effect of the intrinsic curvature $C_{0}$, global oscillations of the axonemal curvature $C_{0}^{\prime}$, and the main dynamic mode $C_{1}$ on the translational and rotational 

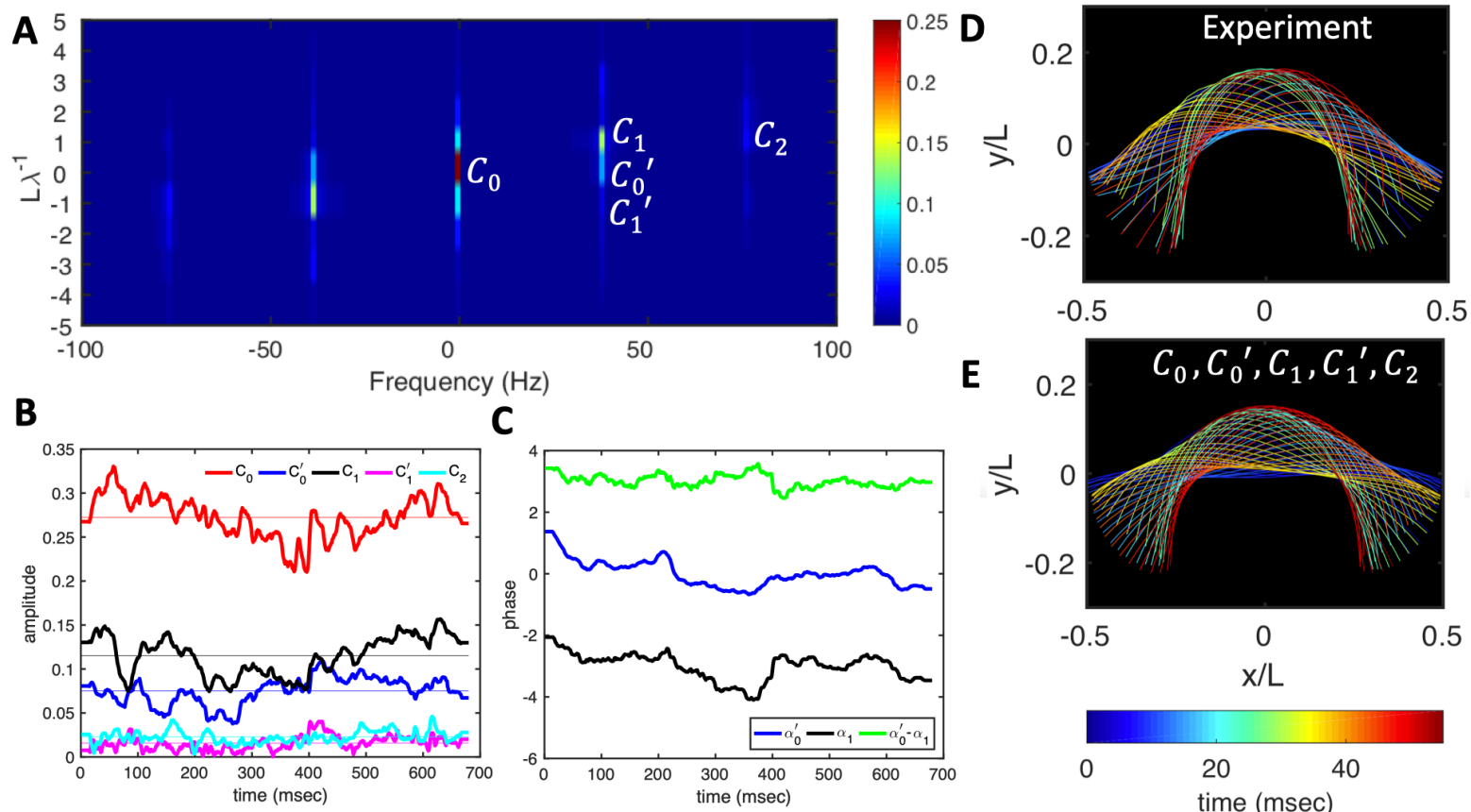

C

$\mathbf{E}$
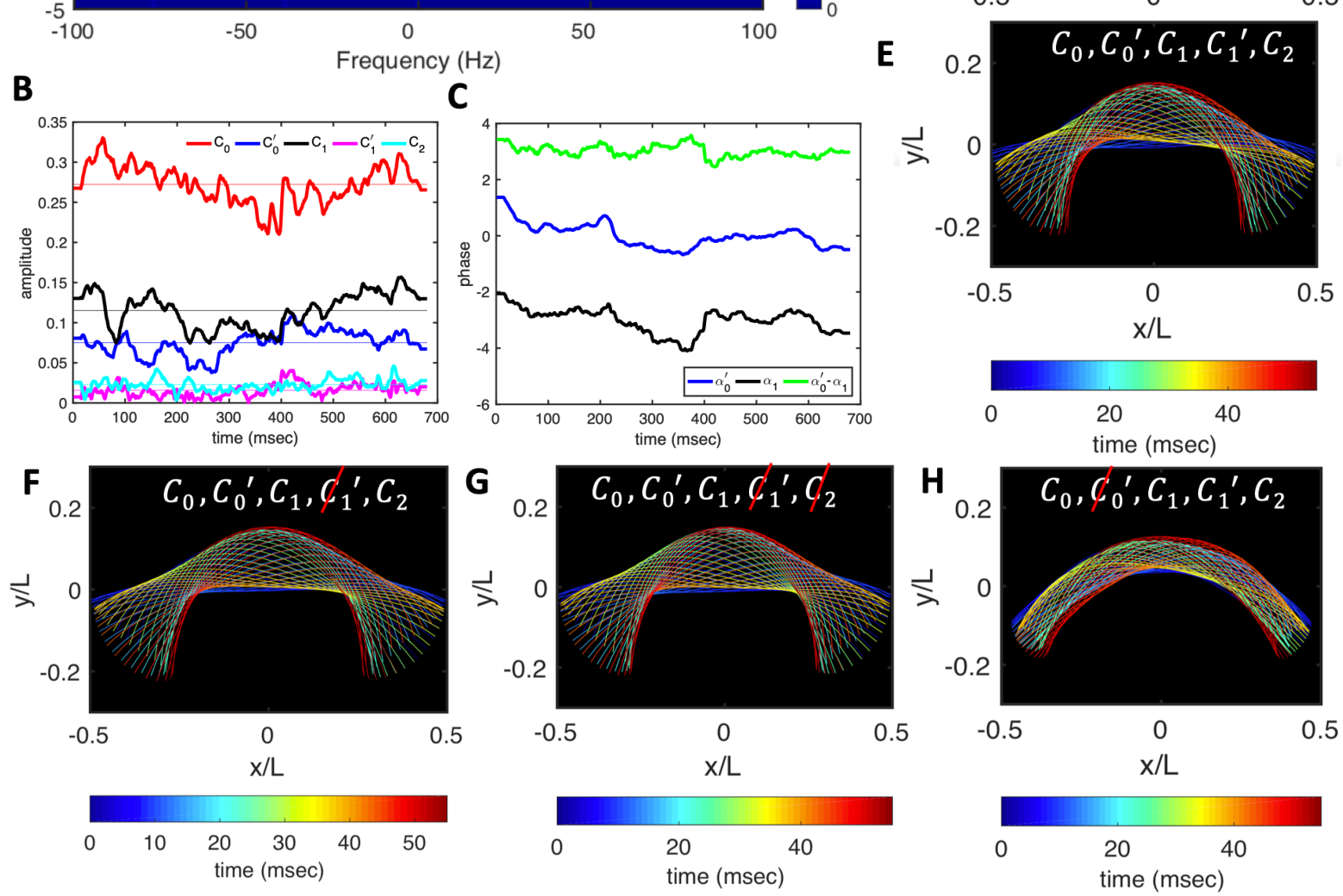

Figure 3. A) The 2D power spectrum of 'windowed' curvature waves shown in Fig. 2D. Dominant modes are highlighted: $C_{0}$ describes the intrinsic curvature, $C_{0}^{\prime}$ refers to the global oscillations of the axonemal curvature, $C_{1}$ and $C_{1}^{\prime}$ are the base-to-tip and tipto-base traveling wave components at the beat frequency $f_{0}=38.24 \mathrm{~Hz}$, and $C_{2}$ is the second harmonic. B) Amplitudes of the dominant modes vary over time. Please note that the second harmonic $C_{2}$ and the back-propagating wave $C_{1}^{\prime}$ have similar amplitudes and both are smaller than $C_{0}^{\prime}$ mode. C) Phase information of $C_{0}^{\prime}$ and $C_{1}$ modes shows that they are almost out of phase $\left(\gamma=\alpha_{0}^{\prime}-\alpha_{1} \sim \pi\right)$. D-E) Experimental axonemal shapes over one beat cycle and the corresponding reconstructed shapes using $C_{0}, C_{0}^{\prime}, C_{1}, C_{1}^{\prime}$ and $C_{2}$ modes. While removing $C_{1}^{\prime}$ mode in panel $\mathrm{F}$ ) or $C_{1}^{\prime}$ and $C_{2}$ modes in panel G) do not significantly impact the reconstructed shapes, eliminating $C_{0}^{\prime}$ mode in panel $\mathrm{H}$ ) shows a strong effect.

velocities of an axonemal-propelled bead, we used the following simplified waveform to describe the dimension-less curvature $C(s, t)$ :

$$
C(s, t) \approx C_{0}+C_{0}^{\prime} \cos \left(\omega_{0} t+\alpha_{0}^{\prime}\right)+C_{1} \cos \left(\omega_{0} t-k s+\alpha_{1}\right) .
$$

Here $\omega_{0}=2 \pi f_{0}$ and the wave number $k=2 \pi / \lambda \sim 2 \pi / L$, where wavelength is found 
to be of the order of axonemal length $L$. Here $\gamma=\alpha_{0}^{\prime}-\alpha_{1}$ is the phase difference between the main traveling wave component and the mode describing the global oscillations of the axonemal curvature. Mode analysis of the exemplary axoneme in Fig. 2B shows that the phase difference $\gamma$ is around $\pi$ and $\bar{C}_{0}^{\prime} / \bar{C}_{1} \sim 0.65$. We emphasize that these numbers are comparable to the corresponding values reported for a free axoneme without any bead in Ref. [26].

Next, we derived analytical forms for translational and rotational velocities of an axonemal-propelled bead in the regime of small $C_{0}, C_{0}^{\prime}$ and $C_{1}$. For our analytical approximations and simulations, we used the resistive-force theory (RFT) which is presented in Sec. 2.3. To check the validity of RFT, we extracted rotational and translational velocities of another exemplary axonemal-driven bead in the supplemental Video 3 and compared the results with velocities obtained by RFT simulations using experimental beat patterns (see Sec. 2.3). This comparison is presented in Fig. S2, which demonstrates a qualitative agreement between RFT simulations and experimental data. We present our analytical approximations in two cases: first, a symmetric bead-axoneme attachment and second, an asymmetric attachment.

3.2.1. Symmetric bead-axoneme attachment. Let us first consider the example where an axoneme is attached from the basal side to a bead, so that the tangent vector at $s=0$ passes through the bead center (Figs. 5A and 7A). To uniquely determine the translational and rotational velocities of the swimmer in dependence of the dimensionless bead radius $r=R / L$, we used the prescribed form of curvature waves in Eq. 14 and imposed the force-free and torque-free constrains in $2 \mathrm{D}$. The drag matrices of bead and axoneme are calculated with respect to the reference point, which is defined as the beadaxoneme contact point (see Sec. 2.4). We approximate analytically the averaged angular and linear velocities in the swimmer-fixed frame, as defined in Fig. 2A, up to the first order in $C_{0}$ and $C_{0}^{\prime}$, and the second order in $C_{1}$. The full expressions are presented in supplemental information, Eqs. S.16- S.18. The results for a bead of size $r=0.1$ and $\eta=\zeta_{\|} / \zeta_{\perp}=0.5$ are as follows:

$$
\begin{aligned}
& \frac{\left\langle\Omega_{z}\right\rangle}{\omega_{0}} \approx-0.24 C_{0} C_{1}^{2}\left(1-2.13 \frac{C_{0}^{\prime}}{C_{1}} \cos \gamma-0.31 \frac{C_{0}^{\prime}}{C_{1}} \sin \gamma\right), \\
& \frac{\left\langle U_{x}\right\rangle}{L \omega_{0}} \approx-0.04 C_{1}^{2}\left(1-1.57 \frac{C_{0}^{\prime}}{C_{1}} \cos \gamma-1.19 \frac{C_{0}^{\prime}}{C_{1}} \sin \gamma\right), \\
& \frac{\left\langle U_{y}\right\rangle}{L \omega_{0}} \approx 0.006 C_{0} C_{1}^{2}\left(1+8.6 \frac{C_{0}^{\prime}}{C_{1}} \cos \gamma-21.15 \frac{C_{0}^{\prime}}{C_{1}} \sin \gamma\right) .
\end{aligned}
$$

We summarize some important properties of Eq. 15. First, without even harmonics, e.g. intrinsic curvature $C_{0}$ or the second harmonic $C_{2}$, the axoneme swims in a straight path with $\left\langle U_{y}\right\rangle=0,\left\langle U_{x}\right\rangle$ proportional to the square of traveling wave component $C_{1}$ [38-40], and the mean rotational velocity $\left\langle\Omega_{z}\right\rangle$ vanishes (see Fig. S3) [24, 25]. Second, the time-symmetric mode $C_{0}^{\prime}$ describing the global oscillations of the axonemal curvature, by itself cannot contribute in propulsion (scallop theorem $[27,41]$ ). However, 
once the traveling wave component $C_{1}$ breaks the time symmetry, it contributes in enhancing the translational and rotational velocities [26]. Third, the phase difference between the axonemal global oscillations and the main traveling wave component $\gamma=\alpha_{0}^{\prime}-\alpha_{1}$, also contributes to the rotational and translational velocities. Last, we note that in comparison to Eqs. S.19-S.21 for a free axoneme without any bead [26], the pre-factors $0.24,0.04$ and 0.006 in Eq. 15 are smaller. For the exemplary axoneme in Fig. 3, we have $\gamma \sim \pi, \bar{C}_{0}^{\prime} / \bar{C}_{1} \sim 0.65$, which further simplifies Eq. 15 to:

$$
\begin{aligned}
& \frac{\left\langle\Omega_{z}\right\rangle}{\omega_{0}} \approx-0.57 C_{0} C_{1}^{2}, \\
& \frac{\left\langle U_{x}\right\rangle}{L \omega_{0}} \approx-0.08 C_{1}^{2}, \\
& \frac{\left\langle U_{y}\right\rangle}{L \omega_{0}} \approx-0.027 C_{0} C_{1}^{2} .
\end{aligned}
$$

It is important to emphasize that the pre-factors in Eq. 16 are at least two times larger than the corresponding numbers with $C_{0}^{\prime}=0$, which highlights the significant effect of the global oscillations of the axonemal curvature in enhancing the translational and rotational velocities [26].

In parallel to our analytical approximations, we also performed numerical simulations given the simplified wave form in Eq. 14 and using RFT with $\eta=0.5$. As shown in Fig. 4, the comparison between numerical simulations and the full analytical approximations presented in Eqs. S.16-S.18, shows a good agreement at small values of $C_{0}, C_{0}^{\prime}$ and $C_{1}$ and deviations at larger values. It is remarkable that while $\left\langle U_{x}\right\rangle$ reduces monotonically with the bead radius $r$, both $\left\langle\Omega_{z}\right\rangle$ and $\left\langle U_{y}\right\rangle$ exhibit nonmonotonic dependence. This anomalous behavior is counter-intuitive, since rotational and translational speeds are expected to decrease with increasing the bead radius.

\subsubsection{Sideways bead attachment contributes to the rotational velocity. In our} experiments, we frequently observed an asymmetric attachment of a bead to an axoneme, i.e. the tangent vector of the axoneme at $s=0$ does not pass through the bead center (see Video 3). This case is schematically shown in Fig. 1A and Figs. 5B-C. Interestingly, our analytical approximations and simulations confirm that this asymmetry is enough to rotate the axoneme and presence of static curvature and second harmonic are not necessary for rotation to occur. For this analysis, we consider the $2 \mathrm{D}$ case where the center of the bead is at position $X_{B}$ and $Y_{B}$ which is measured with respect to the coordinate system defined at the bead-axoneme contact point (Figs. 1A and 2A). Drag matrix of the bead is given in Material and Methods. To focus on the effect of the sideways bead attachment on the axonemal rotational velocity, we set both modes $C_{0}$ and $C_{0}^{\prime}$ to zero and consider a model sperm-like axoneme with only the traveling wave component $C_{1} \neq 0$ in Eq. 14 . We calculate the mean rotational velocity of the axonemaldriven bead by combining the drag matrix of the bead and the axoneme (see Materials 

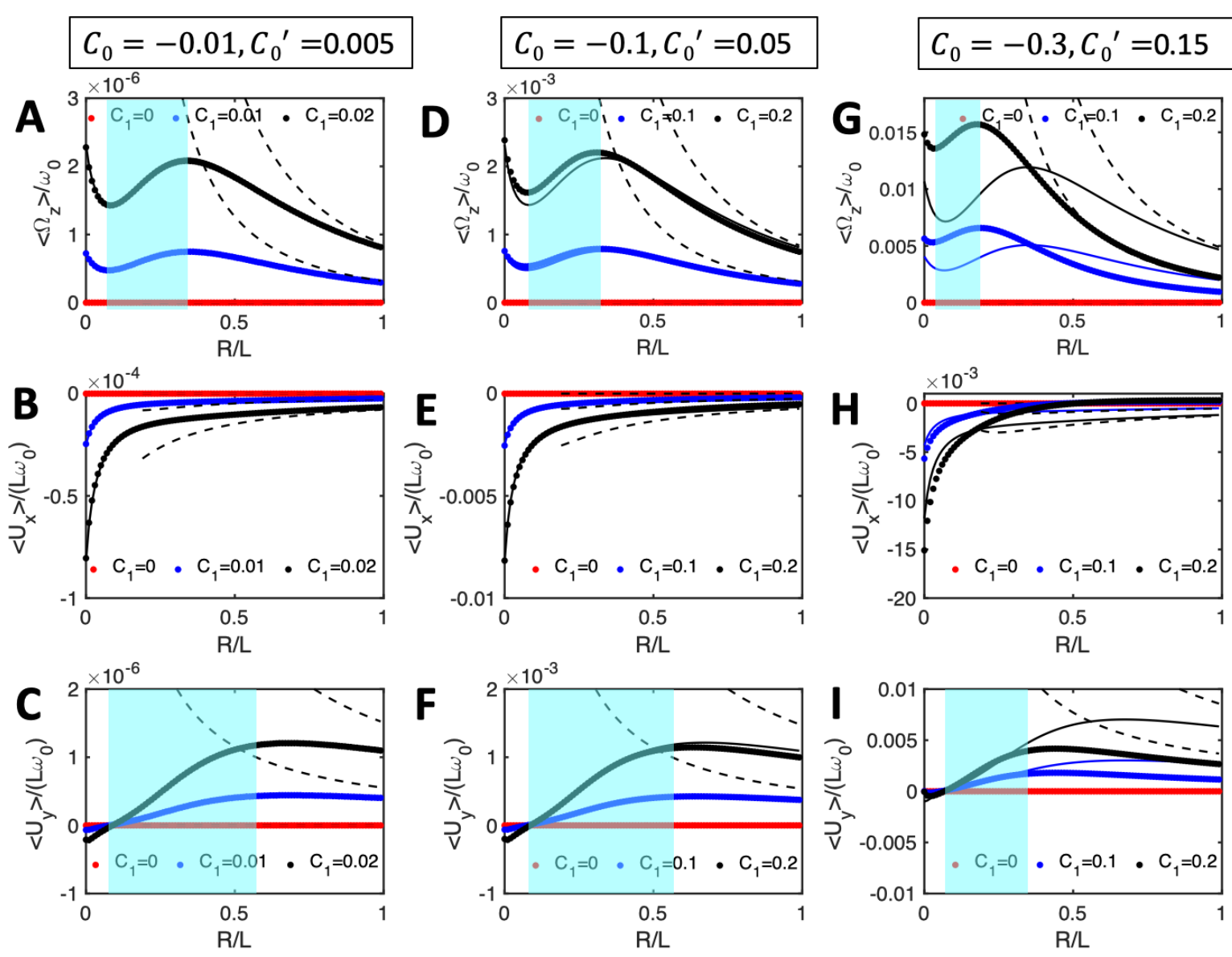

Figure 4. Anomalous flagella-based propulsion speed of a symmetrically attached bead as a function of its dimensionless radius $R / L$. Analytical approximations (continuous lines calculated from Eqs. S.16- S.18) and simulations (dotted points) are performed at different values of $C_{1}$, while the intrinsic curvature of the axoneme and mode describing the global oscillations of axonemal curvature are fixed at $C_{0}=-0.01$ and $C_{0}^{\prime}=0.005$ in $(\mathrm{A}-\mathrm{C}), C_{0}=-0.1$ and $C_{0}^{\prime}=0.05$ in $(\mathrm{D}-\mathrm{F})$, and $C_{0}=-0.3$ and $C_{0}^{\prime}=0.15$ in (G-I). Note that $C_{2}=0$ and $C_{1}^{\prime}=0$ in both simulations and analytical approximations. Areas in cyan color highlight the anomalous propulsion regime. The black dashed curves show the trend expected in the limit of large bead radius $(r=R / L>1)$, as presented in Eqs. S.22- S.24.

and Methods) to arrive at:

$$
\left\langle\Omega_{z}\right\rangle / \omega_{0} \approx \alpha\left(\eta, \zeta_{\perp}^{\prime}, y_{b}, r\right) C_{1}^{2}
$$

where dimensionless quantities are $r=R / L, y_{b}=Y_{B} / L, \eta=\zeta_{\|} / \zeta_{\perp}$ and $\zeta_{\perp}^{\prime}=$ $\zeta_{\perp} / \mu=4 \pi /(\ln (2 L / a)+0.5)$. Here $\mu$ is the viscosity of the ambient water-like fluid and $a=100 \mathrm{~nm}$ is the axonemal radius. Note that the variable $x_{b}^{2}=r^{2}-y_{b}^{2}$ is not an independent variable. For the complete form of the function $\alpha$, see Eq. S.28.

Some important characteristics of Eq. 17 are as follows: First, since even harmonics are set to zero, $\left\langle\Omega_{z}\right\rangle$ is zero for the case of a symmetric bead attachment, i.e. $x_{b}=-R / L$ and $y_{b}=0$. The effect of sideways bead attachment on rotational velocity of the 

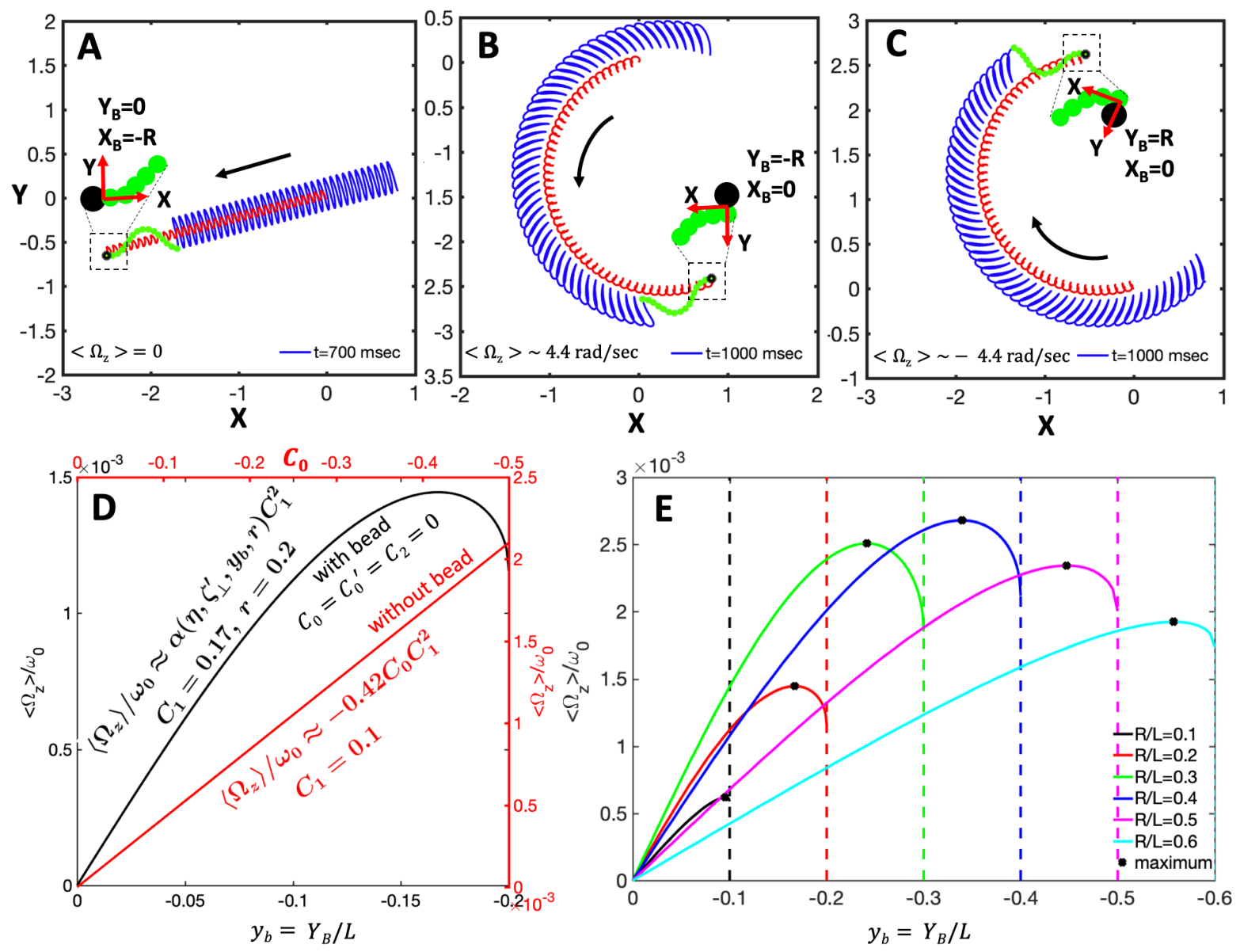

Figure 5. A-C) Asymmetric versus symmetric bead attachment to an axoneme with a beat pattern consisting only of the traveling wave component $C_{1}$. While the axoneme in panel A, to which the bead is symmetrically attached, swims on a straight path (Video 4), the axonemes in panel B (Video 5) and C (Video 6), with asymmetric bead attachment, swim on curved paths. D) Comparison of the effect of the asymmetric bead attachment (in black) as a function of $y_{b}$ versus the effect of the intrinsic curvature $C_{0}$ (in red) on $\left\langle\Omega_{z}\right\rangle$. E) The averaged angular velocity $\left\langle\Omega_{z}\right\rangle$ changes non-monotonically with $y_{b}$ for different bead radii. $X_{B}$ and $Y_{B}$ are the coordinates of the bead center in the swimmer-fixed reference frame. Parameters are $\eta=0.5, \zeta_{\perp}^{\prime}=4.33$ and $C_{1}=0.1$.

axoneme is highlighted in Fig. 5A-C. In these simulations, the model axoneme has only the traveling wave component $C_{1}$ and it swims in a straight path if the bead is attached symmetrically (Fig. $5 \mathrm{~A}$ and Video 4). An asymmetric bead-axoneme attachment causes the axoneme to rotate (Fig. 5B-C and Videos 5-6). Second, $\left\langle\Omega_{z}\right\rangle$ changes non-monotonically with the bead radius as shown in Fig. S4. Note that for an asymmetric attachment of the bead at $y_{b}=-r$, the maximum $\left\langle\Omega_{z}\right\rangle$ occurs at $r=0.4$. Third, a comparison between the effect of the intrinsic curvature (Eq. S.16 with $C_{0}^{\prime}=0$ and $r=0$ ) versus asymmetric bead attachment (Eq. 17 with $\eta=0.5, \zeta_{\perp}^{\prime}=4.33$ and $r=0.2$ ) on rotational velocity shows that the contribution of the asymmetry in the attachment to $\left\langle\Omega_{z}\right\rangle$ is comparable to that of the intrinsic curvature, $C_{0}$, see Fig. 5D. 
Last, as shown in Fig. 5E, the maximum rotational velocity occurs in the vicinity of $y_{b}=-r$.

\subsection{Analysis with experimental waveform}

To confirm that anomalous propulsion regime and the rotation induced by asymmetric cargo attachment is general and not limited to the simplified waveform introduced in Eq. 14, we also used experimental beat patterns of the exemplary axoneme in Fig. 2. We performed RFT simulations to compute translational and rotational velocities of the axoneme-bead swimmer using experimental waveform as an input. We considered both asymmetric and symmetric bead-axoneme attachment. As shown in Fig. 6, the anomalous propulsion regime exist for both symmetric and asymmetric attachments. Depending on the sign of the static curvature of the axoneme $C_{0}$ and the position at which the bead is attached, the asymmetric attachment may contribute to an increase or decrease in the overall mean rotational velocities. For the axoneme in Fig. 6, $C_{0}$ is negative and the sideways bead attachment at $Y_{B}=R$ (Fig. 6A) acts against the rotation induced by the intrinsic curvature $C_{0}$. The opposite happens in Fig. $6 \mathrm{~B}$ where the bead attachment at $Y_{B}=-R$ amplifies the rotational velocity of the axoneme. We also note that the anomalous propulsion regime is more pronounced in panels $\mathrm{B}$ and $\mathrm{C}$ where the
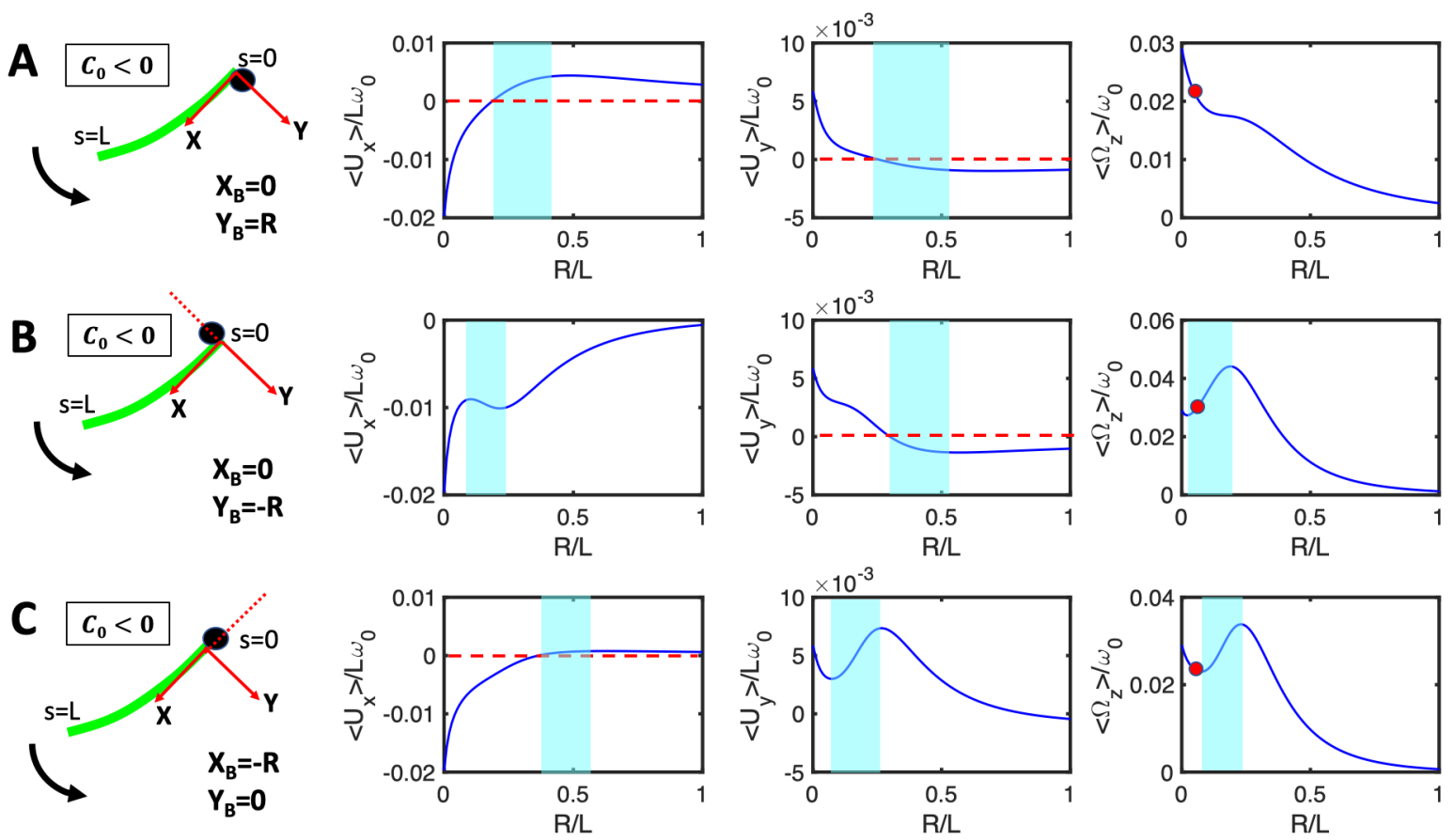

Figure 6. Experimental beat pattern in Fig. 2 are used to calculate translational and rotational velocities of an axonemal-propelled bead attached (A) asymmetrically at $Y_{B}=R, X_{B}=0$ (B) asymmetrically at $Y_{B}=-R, X_{B}=0$ and (C) symmetrically at $Y_{B}=0, X_{B}=-R$. Anomalous propulsion regimes are highlighted in cyan color. Red circles mark the experimental bead size of $R / L \sim 0.05$ and the corresponding rotational velocities. 
bead is attached sideways at $Y_{B}=-R$ or symmetrically at $X_{B}=-R$ and $Y_{B}=0$. Further, in Fig. 6, comparing panels A-C shows that at $R / L \sim 0.5 \mu m / 9.4 \mu m \sim 0.05$ which is close to the experimental bead size, $\left\langle\Omega_{z}\right\rangle / \omega_{0}$ is 0.021 in panel A, 0.029 in panel $\mathrm{B}$ and 0.024 in panel C. Given $f_{0}=38.24 \mathrm{~Hz}$ for the experiment in Fig. 2, we obtain $\left\langle\Omega_{z}\right\rangle / \omega_{0} \sim 0.04$, which is closest to the value of 0.029 in panel $\mathrm{B}$. We attribute this discrepancy to multiple factors: 1) Our simulations are done assuming the validity of RFT with $\eta=\zeta_{\|} / \zeta_{\perp}=0.5$. As shown in Fig. S2, we obtained a qualitative agreement between experimentally measured translational and rotational velocities and RFT simulations. 2) We note that any error in axoneme tracking directly affects the value of the measured curvature waves that are used as experimental input in our analysis.

Finally, to induce a transition from a circular to a straight sperm-like swimming trajectory, we used $0.1 \mathrm{mM}$ Calcium ions to reduce the static curvature of axonemes around 10 times $[19,26,42]$. In the representative example shown in Fig. 7, the static curvature $\kappa_{0}$ is reduced to $-0.03 \mu \mathrm{m}^{-1}$ (see cyan filament in Fig. 7D), and as a result the rotational velocity of the axoneme is decreased in comparison to Fig. 6. In this example, the bead-axoneme attachment appears to be symmetric and we again observe the existence of anomalous propulsion regimes. The experimental value of $\left\langle\Omega_{z}\right\rangle / \omega_{0} \sim 0.008$ (total rotation of $\sim \pi / 2$ in 1299 msec; see Video 7) is comparable to the value of 0.007 which is highlighted by red circles in Fig. 7E-G.

\section{Conclusions}

In this work, we isolated flagella from green algae $C$. reinhardtii by dibucaine protocol and removed the membrane by treatment of detached flagella with non-ionic detergents. The remaining $9+2$ microtubule-based structure which lacks membrane and basal bodies can be attached to a bead and be actuated by an ATP-supplemented reactivation buffer to propel the bead. In our experiments, since beads were incubated with axonemes, the quantity, spacing and location of beads attached to the axonemes were not controlled. Normally, a small fraction of the beads adheres to the axonemes at random sites preferably at the basal or the distal ends; see Videos 8-9 as examples that two axonemes are attached to one bead or two beads adhered to one axoneme.

Isolated axonemes beat with an asymmetric waveform predominantly in $2 \mathrm{D}$, which resembles the forward swimming motion of flagella in intact cells. We extracted the shape of axoneme by gradient vector flow technique and quantitatively described the beating pattern by the dimensionless curvature $C(s, t)$ at time $t$ and at arc-length $s$ along the axonemal length. The power spectrum of curvature waves in $2 \mathrm{D}$ reveals that multiple modes contribute in axonemal curvature. The well-known modes are the static curvature, the main base-to-tip traveling wave component with wavelength $L$ and fundamental beat frequency $f_{0}$, and its higher harmonics. The static component of

curvature defines a circular arc with mean curvature of around $-0.19 \mu \mathrm{m}^{-1}$ and has the dominant contribution in rotational velocity of the axoneme. However, we also observed 

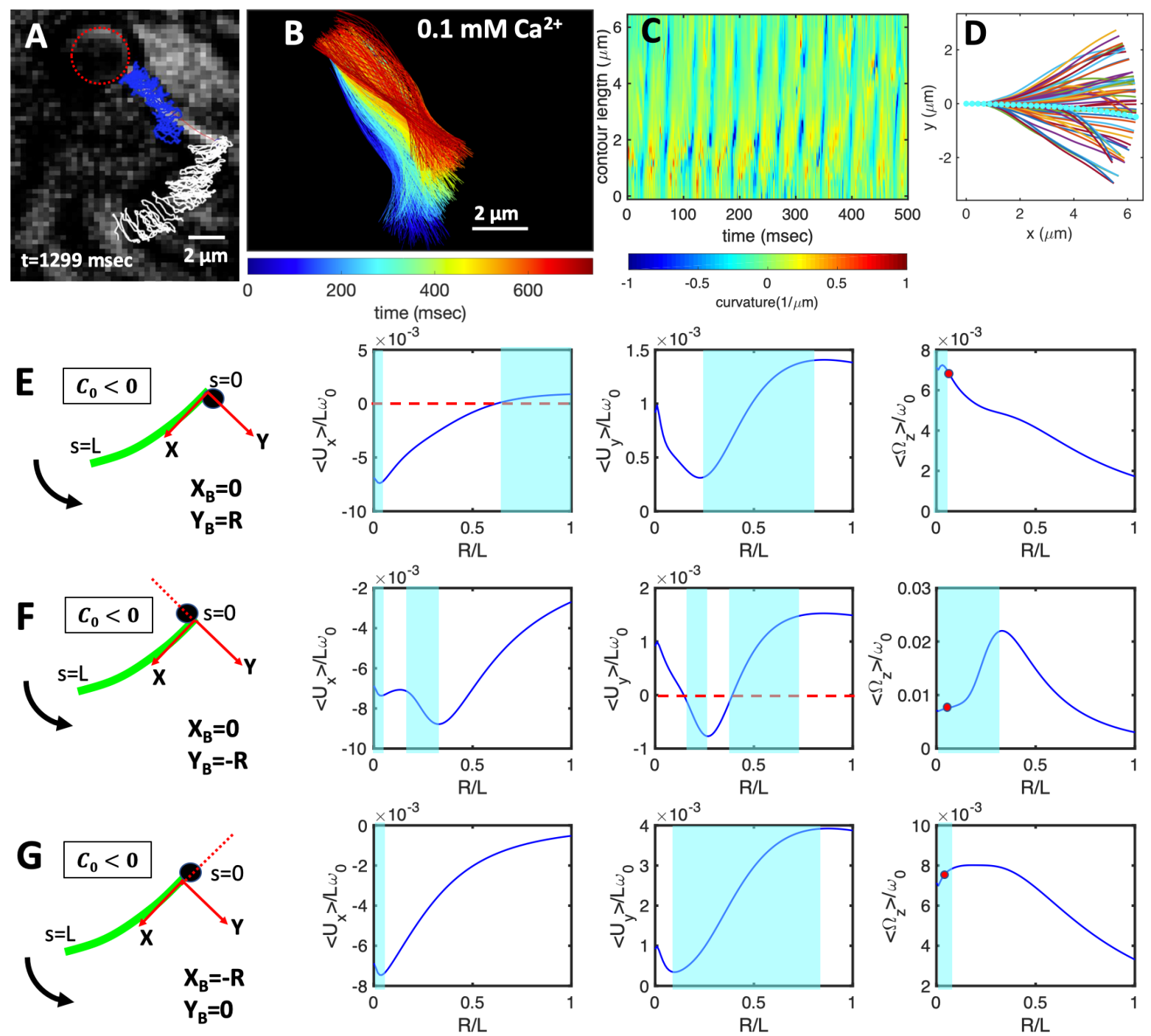

Figure 7. A) An axoneme-driven bead reactivated with $80 \mu \mathrm{M}$ ATP. The reactivation buffer is supplemented with $0.1 \mathrm{mM}$ calcium to reduce the static curvature. The axoneme beats at $24.96 \mathrm{~Hz}$ and the bead-axoneme attachment appears to be symmetric. B) Time-projection of the axonemal beat pattern, C) the base-to-tip propagating curvature waves, and D) axonemal mean shape with static curvature of $\kappa_{0} \sim-0.03$ $\mu \mathrm{m}^{-1}$ (filament with cyan color). Experimental beat pattern shown in panel B are used to calculate the mean translational and rotational velocities of the axonemaldriven bead attached (E) asymmetrically at $Y_{B}=R, X_{B}=0(\mathrm{~F})$ asymmetrically at $Y_{B}=-R, X_{B}=0$ and $(\mathrm{G})$ symmetrically at $Y_{B}=0, X_{B}=-R$. Anomalous propulsion regimes are highlighted in cyan color. Red circles in panels E-G mark the experimental bead radius of $R / L=0.05$ and the corresponding value of 0.007 of $\left\langle\Omega_{z}\right\rangle / \omega_{0}$ (see supplemental Video 7). 
another peak at the beat frequency $f_{0}$ but at infinite wavelength. This mode highlights the global oscillations of the axonemal curvature and can lead to $\sim 2$ times enhancement of translational and rotational velocities of a free axoneme, as we have shown in Ref. [26] or an axonemal-driven bead, as we have discussed in this work.

We approximated the axonemal waveform as the superposition of static curvature $C_{0}$, main traveling wave component $C_{1}$ and the mode describing the global oscillations of the axonemal curvature $C_{0}^{\prime}$. Both the tip-to-base propagating wave component $C_{1}^{\prime}$ and the second harmonic $C_{2}$, have comparable, albeit small, amplitudes compared to $C_{0}, C_{1}$ and $C_{0}^{\prime}$ modes and removing these two modes from the shape analysis does not significantly affect the accuracy of the reconstructed shapes. The simplified waveform composed of $C_{0}, C_{1}$ and $C_{0}^{\prime}$ modes is sufficient to reconstruct the flagellar shapes with good accuracy and allows us to obtain analytical expressions for translational and rotational velocities of an axonemal-propelled bead. The rotational velocity of an axoneme is predominately controlled by its static curvature $C_{0}$ and the time-symmetric mode $C_{0}^{\prime}$ by itself cannot result in propulsion (scallop theorem). However, in the presence of the traveling wave mode $C_{1}$ which breaks the time symmetry, it can enhance the translational and rotational velocities of an axonemal-driven bead with a factor of around 2. The phase difference between modes $C_{0}^{\prime}$ and $C_{1}$ also contributes to translational and rotational velocities. These two modes are typically out of phase with a phase difference of around $\pi$. Importantly, our analysis shows that an anomalous propulsion regime exist where the rotational and translational velocities of an axonemalpropelled bead increases with the bead radius. This anomalous propulsion regime also exists for a model sperm-like swimmer which has a zero intrinsic curvature $C_{0}$, and is propelled by the traveling wave component $C_{1}$ (see Fig. S4). An anomalous cargo transport regime is also reported in Refs. [43,44], where a cargo is propelled by rotation of the helical flagella of bacteria Pseudomonas aeruginosa.

Finally, our analysis shows that asymmetric cargo-axoneme attachment contributes at the same order of magnitude as the intrinsic curvature to the rotational velocity. In other words, a sperm-like beating flagellum without intrinsic curvature and second harmonic swims in a curved trajectory if it is attached sideways to a cargo. In our experiments, since we image the sample in $2 \mathrm{D}$, we are not able to precisely distinguish symmetric versus asymmetric bead-axoneme attachments. In a 2D-projected image, a symmetric bead-axoneme attachment could in reality be an asymmetric one. 3D microscopy techniques, similar to the one used in Ref. [45] are necessary to fully characterize symmetric versus asymmetric axoneme-bead attachment in 3D.

Design and fabrication of micro-scale synthetic swimmers is a challenging task in the growing field of smart drug delivery. Flagella isolated from biological micro-organisms show variety of waveforms and are promising candidates to provide power source for motility by an effective conversion of chemical energy to mechanical work. Our analysis shows an anomalous dependency of the propulsion speed on the cargo size and also highlights the contribution of asymmetric cargo-flagellum attachment in the rotational velocity of the micro-swimmer. This turning mechanism should be taken into account 
in manufacturing bio-inspired synthetic swimmers where a directional targeted motion is critical for delivery of drug-loaded cargoes.

\section{Acknowledgment}

The authors acknowledge J. Molacek, F. Nordsiek, B. Nasouri and Y. Wang for valuable comments and discussions and S. Romanowsky, M. Müller and K. Gunkel for technical assistance. R.A. acknowledge support from the European Union's Horizon 2020 research and innovation programme under grant agreement MAMI No. 766007. A.G. and A.B. acknowledge MaxSynBio Consortium, which is jointly funded by the Federal Ministry of Education and Research of Germany and the Max Planck Society. The authors also thank M. Lorenz and S. Bank at the Göttingen Algae Culture Collection (SAG) for providing the $C$. reinhardtii wild type strain SAG 11-32b.

\section{Author contributions}

A.G. designed the project. R.A. isolated flagella and performed the experiments. R.A., Y.S., S.G.P. and A.G. analyzed the flagella data. A.B. wrote the Matlab code to track the axonemes. A. G. and A.B. performed the theoretical analysis and simulations. A.G. wrote the first draft of the manuscript and all the authors discussed the results and contributed to the revised version.

\section{References}

[1] Gilpin W, Bull M S and Prakash M 2020 Nature Reviews Physics 1-15

[2] Eggenschwiler J T and Anderson K V 2007 Annu. Rev. Cell Dev. Biol. 23 345-373

[3] Faubel R, Westendorf C, Bodenschatz E and Eichele G 2016 Science 353 176-178

[4] Shah A S, Ben-Shahar Y, Moninger T O, Kline J N and Welsh M J 2009 Science 325 1131-1134

[5] Witman G 2009 The Chlamydomonas Sourcebook: Cell Motility and Behavior: Volume 3 vol 3 (Academic press)

[6] Vincensini L, Blisnick T and Bastin P 2011 Biology of the Cell 103 109-130

[7] Goldstein R E 2015 Annual review of fluid mechanics 47 343-375

[8] Goldstein R E, Polin M and Tuval I 2009 Physical review letters 103168103

[9] Wan K Y, Leptos K C and Goldstein R E 2014 Journal of the Royal Society Interface 1120131160

[10] Chen D T, Heymann M, Fraden S, Nicastro D and Dogic Z 2015 Biophysical journal 109 2562-2573

[11] Nicastro D, Schwartz C, Pierson J, Gaudette R, Porter M E and McIntosh J R 2006 Science 313 944-948

[12] Lin J and Nicastro D 2018 Science $\mathbf{3 6 0}$

[13] King S M 2018 Composition and assembly of axonemal dyneins Dyneins: Structure, biology and disease (Elsevier) pp 162-201

[14] Bower R, Tritschler D, VanderWaal K, Perrone C A, Mueller J, Fox L, Sale W S and Porter M 2013 Molecular biology of the cell 24 1134-1152

[15] Pigino G, Bui K H, Maheshwari A, Lupetti P, Diener D and Ishikawa T 2011 Journal of Cell Biology $195673-687$

[16] Yang P, Diener D R, Yang C, Kohno T, Pazour G J, Dienes J M, Agrin N S, King S M, Sale W S, Kamiya R et al. 2006 Journal of cell science 119 1165-1174 
[17] Heuser T, Raytchev M, Krell J, Porter M E and Nicastro D 2009 Journal of Cell Biology 187 921-933

[18] Luck D, Piperno G, Ramanis Z and Huang B 1977 Proceedings of the National Academy of Sciences $743456-3460$

[19] Bessen M, Fay R B and Witman G B 1980 The Journal of Cell Biology 86 446-455

[20] Hyams J S and Borisy G G 1978 Journal of Cell Science 33 235-253

[21] Geyer V F, Sartori P, Friedrich B M, Jülicher F and Howard J 2016 Current Biology 26 1098-1103

[22] Sartori P, Geyer V F, Scholich A, Jülicher F and Howard J 2016 Elife 5 e13258

[23] Geyer V 2013 Characterization of the flagellar beat of the single cell green alga Chlamydomonas reinhardtii Ph.D. thesis Sächsische Landesbibliothek-Staats-und Universitätsbibliothek Dresden

[24] Saggiorato G, Alvarez L, Jikeli J F, Kaupp U B, Gompper G and Elgeti J 2017 Nature communications 8 1-9

[25] Gong A, Rode S, Kaupp U B, Gompper G, Elgeti J, Friedrich B M and Alvarez L 2020 Philosophical Transactions of the Royal Society B $\mathbf{3 7 5} 20190149$

[26] Gholami A, Ahmad R, Bae A, Pumir A and Bodenschatz E 2021 bioRxiv

[27] Purcell E M 1977 American journal of physics 45 3-11

[28] Gray J and Hancock G 1955 Journal of Experimental Biology 32 802-814

[29] Johnson R and Brokaw C 1979 Biophysical journal 25 113-127

[30] Witman G B 1986 Isolation of chlamydomonas flagella and flagellar axonemes Meth. Enzymology vol 134 (Elsevier) pp 280-290

[31] Alper J, Geyer V, Mukundan V and Howard J 2013 Reconstitution of flagellar sliding Meth. Enzymology vol 524 (Elsevier) pp 343-369

[32] Xu C and Prince J L 1997 Gradient vector flow: A new external force for snakes IEEE Proc. CVPR (IEEE) pp 66-71 ISBN 0-8186-7822-4

[33] Xu C and Prince J L 1998 IEEE Transactions on image processing 7 359-369

[34] Lauga E and Powers T R 2009 Reports on Progress in Physics 72096601

[35] Keller J B and Rubinow S 1976 Biophysical Journal 16 151-170

[36] Leach J, Mushfique H, Keen S, Di Leonardo R, Ruocco G, Cooper J and Padgett M 2009 Physical Review E 79026301

[37] Ahmad R, Kleineberg C, Nasirimarekani V, Su Y J, Goli Pozveh S, Bae A, Sundmacher K, Bodenschatz E, Guido I, Vidakovic-Koch T et al. 2021 ACS Synthetic Biology

[38] Shapere A and Wilczek F 1987 Physical Review Letters 582051

[39] Lauga E 2007 Physical Review E 75041916

[40] Friedrich B M, Riedel-Kruse I H, Howard J and Jülicher F 2010 Journal of Experimental Biology $2131226-1234$

[41] Lauga E 2011 Soft Matter 7 3060-3065

[42] Omoto C and Brokaw C 1985 Cell motility 5 53-60

[43] Prakash P, Abdulla A Z, Singh V and Varma M 2019 Physical Review E 100062609

[44] Prakash P, Abdulla A, Singh V and Varma M 2020 Soft Matter 16 9499-9505

[45] Mojiri S, Isbaner S, Mühle S, Jang H, Bae A J, Gregor I, Gholami A and Enderlein J 2021 Biomedical Optics Express 12 3169-3180

\section{Supplemental Information}

\subsection{Drag matrix of a bead in $3 D$}

Let's fix a couple points $P$ and $P^{\prime}$ on or in a rigid body (see Fig. S1). The distance between these points remains constant. Furthermore, if we attach two parallel vectors at $P$ and $P^{\prime}$, they would remain parallel under movement. Since this is the case, it follows that $\vec{\Omega}_{P}=\vec{\Omega}_{P^{\prime}}$, so we will drop the subscript and call it $\Omega$. 

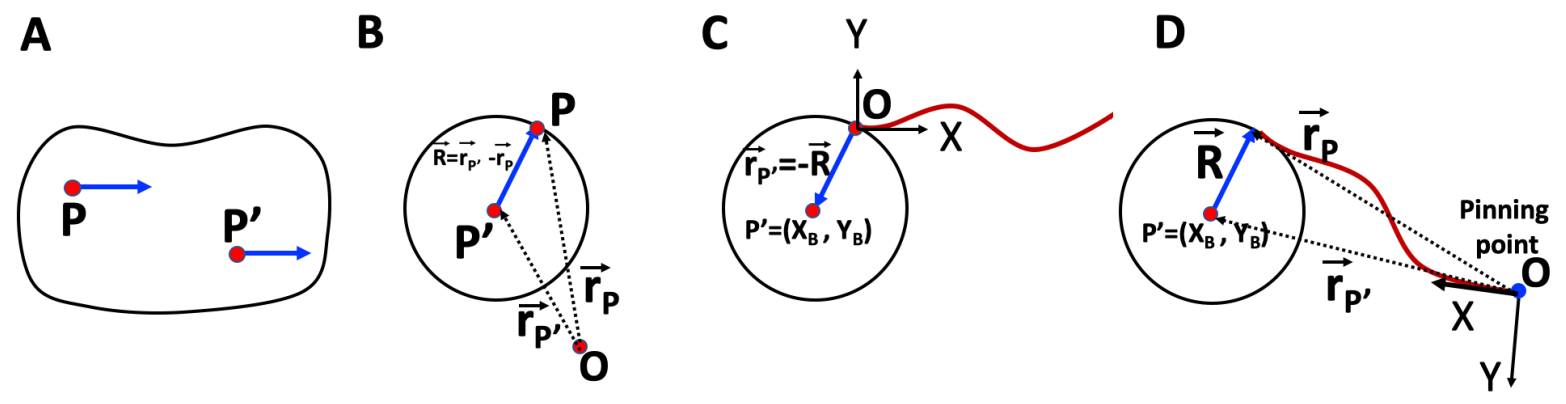

Figure S1. Schematic presentation of the set up and the coordinate system.

Let us denote the positions of $P$ and $P^{\prime}$ by $\vec{r}_{P}$ and $\vec{r}_{P^{\prime}}$. The distance $\left|\vec{r}_{P^{\prime}}-\vec{r}_{P}\right|$ is constant, but the orientation changes:

$$
\vec{V}_{P^{\prime}}-\vec{V}_{P}=\frac{d}{d t}\left(\vec{r}_{P^{\prime}}-\vec{r}_{P}\right)=-\left(\vec{r}_{P^{\prime}}-\vec{r}_{P}\right) \times \vec{\Omega}
$$

Next, suppose we apply a distribution of forces $\vec{f}_{i}$ at $\vec{r}_{i}$ as $\vec{F}=\Sigma_{i} \vec{f}_{i}$ which does not depend on $P$ and $P^{\prime}$. The force moments (i.e. torques) depend on $P$ and $P^{\prime}$ :

$$
\vec{\tau}_{P}=\Sigma_{i}\left(\vec{r}_{i}-\vec{r}_{P}\right) \times \vec{f}_{i} \quad \text { and } \quad \vec{\tau}_{P^{\prime}}=\Sigma_{i}\left(\vec{r}_{i}-\vec{r}_{P}\right) \times \vec{f}_{i}
$$

and the difference gives:

$$
\vec{\tau}_{P^{\prime}}-\vec{\tau}_{P}=-\left(\vec{r}_{P^{\prime}}-\vec{r}_{P}\right) \times \vec{F}
$$

Now let us define the problem we wish to solve: What are the force $\vec{F}=$ $\left(F_{x}, F_{y}, F_{z}\right)^{T}$ and torque $\vec{\tau}=\left(\tau_{x}, \tau_{y}, \tau_{z}\right)^{T}$ must we apply at point $P$ on the bead's surface to counteract the drag force and make the bead move with translational velocity $\vec{V}=\vec{V}_{P}$ and angular velocity $\vec{\Omega}$ ? Let us denote the center of the bead as $P^{\prime}$ and define $\vec{R}=\vec{r}_{P}-\vec{r}_{P^{\prime}}$. This problem would have been much simpler if we were asked what force $\vec{F}^{\prime}$ and torque $\vec{\tau}^{\prime}$ needs to be applied at the bead's center to counteract drag:

$$
\left(\begin{array}{c}
\vec{F}^{\prime} \\
\vec{\tau}^{\prime}
\end{array}\right)=\left(\begin{array}{cc}
\nu_{T} \mathbf{I} & \mathbf{O} \\
\mathbf{O} & \nu_{R} \mathbf{I}
\end{array}\right)\left(\begin{array}{l}
\vec{V}^{\prime} \\
\overrightarrow{\Omega^{\prime}}
\end{array}\right)
$$

Here $\nu_{T}=6 \pi R \mu$ and $\nu_{R}=8 \pi R^{3} \mu / 3$ and

$$
\mathbf{I}=\left(\begin{array}{lll}
1 & 0 & 0 \\
0 & 1 & 0 \\
0 & 0 & 1
\end{array}\right) \quad \text { and } \quad \mathbf{O}=\left(\begin{array}{lll}
0 & 0 & 0 \\
0 & 0 & 0 \\
0 & 0 & 0
\end{array}\right)
$$

Now we use what we learned about rigid body motion in Eq. S.1:

$$
\left(\begin{array}{c}
\overrightarrow{V^{\prime}} \\
\vec{\Omega}^{\prime}
\end{array}\right)=\left(\begin{array}{c}
\vec{V}-\left(\begin{array}{c}
\overrightarrow{r^{\prime}}-\vec{r} \\
\vec{\Omega}
\end{array}\right) \times \Omega \\
\vec{\Omega}
\end{array}\right)=\left(\begin{array}{c}
\vec{V}+\vec{R} \times \Omega \\
\vec{\Omega}
\end{array}\right)=\left(\begin{array}{cc}
\mathbf{I} & \mathcal{R} \\
0 & \mathbf{I}
\end{array}\right)\left(\begin{array}{l}
\vec{V} \\
\vec{\Omega}
\end{array}\right)
$$


where we have defined:

$$
\mathcal{R}=\left(\begin{array}{ccc}
0 & R_{z} & R_{y} \\
R_{z} & 0 & -R_{x} \\
-R_{y} & R_{x} & 0
\end{array}\right)=\left(\begin{array}{ccc}
0 & -z+z^{\prime} & y-y^{\prime} \\
z-z^{\prime} & 0 & -x+x^{\prime} \\
-y+y^{\prime} & x-x^{\prime} & 0
\end{array}\right) .
$$

Recall that $x, y, z$ are the coordinates point of the flagella-bead contact point and $x^{\prime}, y^{\prime}, z^{\prime}$ are the coordinates of the the bead center. Similarly, we have that:

$$
\left(\begin{array}{c}
\vec{F}^{\prime} \\
\vec{\tau}^{\prime}
\end{array}\right)=\left(\begin{array}{c}
\vec{F} \\
\vec{\tau}-\left(\overrightarrow{r^{\prime}}-\vec{r}\right) \times \vec{F}
\end{array}\right)=\left(\begin{array}{c}
\vec{F} \\
\vec{\tau}+\vec{R} \times \vec{F}
\end{array}\right)=\left(\begin{array}{cc}
\mathbf{I} & \mathbf{0} \\
\mathcal{R} & \mathbf{I}
\end{array}\right)\left(\begin{array}{l}
\vec{F} \\
\vec{\tau}
\end{array}\right)
$$

so the Eq. S.4 becomes:

$$
\left(\begin{array}{cc}
\mathbf{I} & \mathbf{0} \\
\mathcal{R} & \mathbf{I}
\end{array}\right)\left(\begin{array}{l}
\vec{F} \\
\vec{\tau}
\end{array}\right)=\left(\begin{array}{cc}
\nu_{T} \mathbf{I} & \mathbf{O} \\
\mathbf{O} & \nu_{R} \mathbf{I}
\end{array}\right)\left(\begin{array}{cc}
\mathbf{I} & \mathcal{R} \\
0 & \mathbf{I}
\end{array}\right)\left(\begin{array}{l}
\vec{V} \\
\vec{\Omega}
\end{array}\right)
$$

Multiplying both side by $\left(\begin{array}{cc}\mathbf{I} & \mathbf{O} \\ -\mathcal{R} & \mathbf{I}\end{array}\right)$ and calculating the products of matrices yields:

$$
\left(\begin{array}{l}
\vec{F} \\
\vec{\tau}
\end{array}\right)=\left(\begin{array}{cc}
\nu_{T} \mathbf{I} & \nu_{T} \mathcal{R} \\
-\nu_{T} \mathcal{R} & \nu_{R} \mathbf{I}-\nu_{T} \mathcal{R}^{2}
\end{array}\right)\left(\begin{array}{l}
\vec{V} \\
\vec{\Omega}
\end{array}\right)
$$

where

$$
\begin{aligned}
\mathcal{R}^{2} & =\left(\begin{array}{ccc}
0 & -R_{z} & R_{y} \\
R_{z} & 0 & -R_{x} \\
-R_{y} & R_{x} & 0
\end{array}\right)\left(\begin{array}{ccc}
0 & -R_{z} & R_{y} \\
R_{z} & 0 & -R_{x} \\
-R_{y} & R_{x} & 0
\end{array}\right) \\
& =\left(\begin{array}{ccc}
-R_{y}^{2}-R_{z}^{2} & R_{x} R_{y} & R_{x} R_{z} \\
R_{x} R_{y} & -R_{x}^{2}-R_{z}^{2} & R_{y} R_{z} \\
R_{x} R_{z} & R y R_{z} & -R_{x}^{2}-R_{y}^{2}
\end{array}\right)
\end{aligned}
$$

Possibly noteworthy is that:

$$
\begin{aligned}
\mathcal{R}^{2}+R^{2} \mathbf{I} & =\left(\begin{array}{ccc}
-R_{y}^{2}-R_{z}^{2} & R_{x} R_{y} & R_{x} R_{z} \\
R_{x} R_{y} & -R_{x}^{2}-R_{z}^{2} & R_{y} R_{z} \\
R_{x} R_{z} & R_{y} R_{z} & -R_{x}^{2}-R_{y}^{2}
\end{array}\right)+\left(\begin{array}{ccc}
R^{2} & 0 & 0 \\
0 & R^{2} & 0 \\
0 & 0 & R^{2}
\end{array}\right) \\
& =\left(\begin{array}{ccc}
R_{x}^{2} & R_{x} R_{y} & R_{x} R_{z} \\
R_{x} R_{y} & R_{y}^{2} & R_{y} R_{z} \\
R_{x} R_{z} & R_{y} R_{z} & R_{z}^{2}
\end{array}\right)=\vec{R} \vec{R}^{T}
\end{aligned}
$$

For the motion in 2D (x-y) plane, we are only interested in $F_{x}, F_{y}$ and $\tau_{z}$ as a function of $V_{x}, V_{y}$ and $\Omega_{z}$. Here we also assume $R_{z}=0$, so we are only interested in components 
1,2 , and 6 :

$$
\begin{aligned}
\left(\begin{array}{c}
F_{x} \\
F_{y} \\
\tau_{z}
\end{array}\right)= & \left(\begin{array}{ccc}
\nu_{T} & 0 & \nu_{T} \mathcal{R}_{13} \\
0 & \nu_{T} & \nu_{T} \mathcal{R}_{23} \\
-\nu_{T} \mathcal{R}_{31} & -\nu_{T} \mathcal{R}_{32} & \nu_{R}-\nu_{T} \mathcal{R}_{33}^{2}
\end{array}\right)\left(\begin{array}{c}
V_{x} \\
V_{y} \\
\Omega_{z}
\end{array}\right) \\
= & \left(\begin{array}{ccc}
\nu_{T} & 0 & \nu_{T} R_{y} \\
0 & \nu_{T} & -\nu_{T} R_{x} \\
\nu_{T} R_{y} & -\nu_{T} R_{x} & \nu_{R}+R^{2} \nu_{T}
\end{array}\right)\left(\begin{array}{c}
V_{x} \\
V_{y} \\
\Omega_{z}
\end{array}\right)
\end{aligned}
$$

Up until now, we haven't actually specified where our origin is. Let's set the origin to be at point $P$ on the surface of the bead $\left(\vec{r}_{P}=0\right)$. We will choose $X$ to point tangent to the flagella at the point of attachment, and let the coordinates of the center of the bead be located at $\vec{r}_{P^{\prime}}=\left(X_{B}, Y_{B}, 0\right)$. Note that $X_{B} \leq 0$ and $-R \leq Y_{B} \leq R$. Also note that $\vec{R}=-\vec{r}_{P^{\prime}}$, so the force equation reads:

$$
\left(\begin{array}{c}
F_{x} \\
F_{y} \\
\tau_{z}
\end{array}\right)=\left(\begin{array}{ccc}
\nu_{T} & 0 & -\nu_{T} Y_{B} \\
0 & \nu_{T} & \nu_{T} X_{B} \\
-\nu_{T} Y_{B} & \nu_{T} X_{B} & \nu_{R}+\nu_{T} R^{2}
\end{array}\right)\left(\begin{array}{c}
V_{x} \\
V_{y} \\
\Omega_{z}
\end{array}\right) .
$$

Note that setting $Y_{B} \neq 0$ allows us to handle the case where the flagella is not attached normal to the bead.

\subsection{Rotational and translational velocities of an axoneme attached symmetrically to a bead}

We used the simplified wave form given by Eq. 14 to calculate translational and rotational velocities of a freely swimming axoneme attached symmetrically from basal end to a bead of radius $r=R / L$. For simplicity, the amplitude of second harmonic $C_{2}$ is set to zero. In the limit of small $C_{0}=\kappa_{0} L /(2 \pi), C_{0}^{\prime}=\kappa_{0}^{\prime} L /(2 \pi)$ and $C_{1}=\kappa_{1} L /(2 \pi)$, we calculate rotational and translational velocities of the swimmer from Eq. 4 by calculating the propulsive forces and inverting the drag matrix $\mathcal{D}$ (see sections 2.3-2.4). We then take average over one beating cycle to obtain:

$$
\begin{aligned}
\frac{\left\langle\Omega_{z}\right\rangle}{\omega_{0}} \approx & \frac{7 C_{0} C_{1} \zeta_{\perp}^{\prime}}{5 \pi^{3}\left(7 \eta \zeta_{\perp}^{\prime}+96 \pi r\right)\left(73728 \pi^{2} r^{4}+2688 \zeta_{\perp}^{\prime} \pi(r(5 r+3)+1) r+49 \zeta_{\perp}^{\prime 2}\right)^{2}}\left(5 C_{1}(24461180928\right. \\
& \times(\eta-1) \pi^{8} r^{7}+42467328 \pi^{6}\left(-720 \eta+32(9 \eta-11) \pi^{2}+7(\eta-1)(2 \eta+9) \zeta_{\perp}^{\prime} \pi+240\right) r^{6} \\
& +3096576 \zeta_{\perp}^{\prime} \pi^{5}\left(-24 \eta(26 \eta+67)+16(\eta(8 \eta+31)-47) \pi^{2}+35(\eta-1) \zeta_{\perp}^{\prime} \pi+552\right) r^{5} \\
& +258048 \zeta_{\perp}^{\prime} \pi^{4}\left(288 \pi\left(4 \pi^{2} \eta-21 \eta-4 \pi^{2}+9\right)+7 \zeta_{\perp}^{\prime}(-3 \eta(65 \eta+23)+(\eta(8 \eta+67)\right. \\
& \left.\left.-83) \pi^{2}+24\right)\right) r^{4}+2688 \zeta_{\perp}^{\prime} \pi^{3}\left(-735(\eta(9 \eta-4)+1) \zeta_{\perp}^{\prime 2}+14112(\eta-1) \pi^{3} \zeta_{\perp}^{\prime}-6048\right. \\
& \times(\eta+3)(3 \eta-1) \pi \zeta_{\perp}^{\prime}+18432(\eta-1) \pi^{4}+\left(245\left(2 \eta^{2}+\eta-3\right) \zeta_{\perp}^{\prime 2}-27648(5 \eta-3)\right) \pi^{2} \\
& +82944(2 \eta-1)) r^{3}+56448 \zeta_{\perp}^{\prime 2} \pi^{2}\left(112 \pi^{4}(\eta-1)+7(\eta+2) \zeta_{\perp}^{\prime} \pi^{3}(\eta-1)-24(\eta(4 \eta+31)\right. \\
& \left.-17) \pi^{2}+288(\eta+2)(2 \eta-1)+21 \eta(1-5 \eta) \zeta_{\perp}^{\prime} \pi\right) r^{2}+32928 \zeta_{\perp}^{\prime 3} \pi\left(7 \pi^{4}(\eta-1)-3(\eta(13 \eta\right. \\
& \left.\left.+5)-6) \pi^{2}+36\left(4 \eta^{2}-1\right)\right) r-2401 \zeta_{\perp}^{\prime 4}\left(9\left(-8+\pi^{2}\right) \eta^{2}-\left(-36+\pi^{4}\right) \eta+\pi^{4}-3 \pi^{2}\right)\right) \\
& +C_{0}^{\prime} \pi^{2}\left(-33973862400(\eta-1) \pi^{6}\left(3+\pi^{2}\right) r^{7}-7077888 \pi^{4}\left(768 \pi^{4}(3 \eta-4)+35(\eta-1)\right.\right.
\end{aligned}
$$




$$
\begin{aligned}
& \left.\times(4 \eta+11) \zeta_{\perp}^{\prime} \pi^{3}+960(13 \eta-9) \pi^{2}-1440(13 \eta-9)+105(\eta-1)(8 \eta+31) \zeta_{\perp}^{\prime} \pi\right) r^{6} \\
& -516096 \zeta_{\perp}^{\prime} \pi^{3}\left(-720(2 \eta+7)(13 \eta-9)+64(\eta(16 \eta+23)-68) \pi^{4}+175(\eta-1) \zeta_{\perp}^{\prime} \pi^{3}\right. \\
& \left.+480(3 \eta(8 \eta+23)-77) \pi^{2}+525(\eta-1)(4 \eta+3) \zeta_{\perp}^{\prime} \pi\right) r^{5}+86016 \zeta_{\perp}^{\prime} \pi^{2}\left(2 8 8 \pi \left(-150 \pi^{2}(\eta-1)\right.\right. \\
& \left.+2(2 \eta-1) \pi^{4}+45(13 \eta-9)\right)+7 \zeta_{\perp}^{\prime}\left(45(7 \eta+5)(13 \eta-9)+4(\eta(4 \eta-37)+55) \pi^{4}\right. \\
& \left.\left.+15(\eta(33-146 \eta)+97) \pi^{2}\right)\right) r^{4}+896 \zeta_{\perp}^{\prime} \pi\left(11025 \eta(13 \eta-9) \zeta_{\perp}^{\prime 2}+1008(\eta(8 \eta-11)+11) \pi^{5} \zeta_{\perp}^{\prime}\right. \\
& -15120(\eta-1)(20 \eta+19) \pi^{3} \zeta_{\perp}^{\prime}+90720(\eta+1)(13 \eta-9) \pi \zeta_{\perp}^{\prime}-2\left(245(\eta(\eta+2)-5) \zeta_{\perp}^{\prime 2}\right. \\
& \left.+13824(22 \eta-21)) \pi^{4}-15\left(245(\eta-1)(4 \eta+3) \zeta_{\perp}^{\prime 2}-27648(13 \eta-9)\right) \pi^{2}\right) r^{3}-9408 \zeta_{\perp}^{\prime 2} \\
& \times \pi\left(-720(4 \eta+5) \pi(13 \eta-9)+7(\eta(2 \eta-1)-5) \zeta_{\perp}^{\prime} \pi^{4}+96(\eta(44 \eta+31)-69) \pi^{3}\right. \\
& \left.+105(\eta-1)(4 \eta+5) \zeta_{\perp}^{\prime} \pi^{2}+630 \eta(9-13 \eta) \zeta_{\perp}^{\prime}\right) r^{2}-32928 \zeta_{\perp}^{\prime 3} \pi\left((\eta(76 \eta-5)-59) \pi^{2}\right. \\
& \left.-15(5 \eta+1)(13 \eta-9)) r+2401 \zeta_{\perp}^{\prime 4}\left(15 \eta(13 \eta-9)+(9 \eta(1-2 \eta)+5) \pi^{2}\right)\right) \cos \left(2\left(\alpha_{0}-\alpha_{1}\right) \pi\right) \\
& -96 C_{0}^{\prime} \pi^{4} r\left(70778880(\eta-1) \pi^{3}\left(-15+16 \pi^{2}\right) r^{6}+368640 \pi^{2}\left(96 \pi\left(17 \pi^{2} \eta-39 \eta-21 \pi^{2}+27\right)\right.\right. \\
& \left.+7(\eta-1) \zeta_{\perp}^{\prime}\left(2(7 \eta+19) \pi^{2}-15(2 \eta+5)\right)\right) r^{5}+26880 \zeta_{\perp}^{\prime} \pi\left(48 \pi^{3}(\eta(18 \eta+37)-71)\right. \\
& \left.+35(\eta-1)(\eta+3) \zeta_{\perp}^{\prime} \pi^{2}-525(\eta-1) \eta \zeta_{\perp}^{\prime}-144(\eta(26 \eta+93)-83) \pi\right) r^{4}+2688 \zeta_{\perp}^{\prime} \pi\left(9 6 \pi \left(29 \pi^{2} \eta\right.\right. \\
& \left.\left.-615 \eta-27 \pi^{2}+495\right)+35 \zeta_{\perp}^{\prime}\left(3 \eta(43-111 \eta)+(\eta(19 \eta+21)-48) \pi^{2}+60\right)\right) r^{3} \\
& +56 \zeta_{\perp}^{\prime}\left(-7350 \eta(2 \eta-1) \zeta_{\perp}^{\prime 2}+336(\eta+1)(29 \eta-27) \pi^{3} \zeta_{\perp}^{\prime}-2520(\eta(82 \eta-29)-21) \pi \zeta_{\perp}^{\prime}\right. \\
& \left.+4608(7 \eta-6) \pi^{4}+5\left(245\left(\eta^{2}-1\right) \zeta_{\perp}^{\prime 2}-13824(7 \eta-6)\right) \pi^{2}\right) r^{2}+98 \zeta_{\perp}^{\prime 2}\left(48 \pi^{3}(\eta(28 \eta-11)-9)\right. \\
& \left.-720 \pi(\eta(28 \eta-11)-9)+7(\eta(43 \eta-24)-15) \zeta_{\perp}^{\prime} \pi^{2}+105 \eta(21-37 \eta) \zeta_{\perp}^{\prime}\right) r \\
& \left.\left.+343 \eta(13 \eta-9) \zeta_{\perp}^{\prime 3}\left(-15+\pi^{2}\right)\right) \sin \left(2\left(\alpha_{0}-\alpha_{1}\right) \pi\right)\right) \\
& \frac{\left\langle U_{x}\right\rangle}{L \omega_{0}} \approx-\frac{7 C_{1} \zeta_{\perp}^{\prime}}{6 \pi^{2}\left(49 \zeta_{\perp}^{\prime 2}+2688 \pi \zeta_{\perp}^{\prime}(r(5 r+3)+1) r+73728 \pi^{2} r^{4}\right)\left(7 \eta \zeta_{\perp}^{\prime}+96 \pi r\right)}\left(\pi^{2} C_{0}^{\prime} \cos \left(2 \pi\left(\alpha_{0}-\alpha_{1}\right)\right)\right. \\
& \times\left(-49\left(\left(\pi^{2}-9\right) \eta+3\right) \zeta_{\perp}^{\prime 2}+672 \pi \zeta_{\perp}^{\prime} r\left(20\left(6+\pi^{2}\right)(\eta-1) r^{2}+6\left(9+\pi^{2}\right)(\eta-1) r\right.\right. \\
& \left.\left.+18 \eta-\pi^{2}-12\right)+147456 \pi^{2}\left(3+\pi^{2}\right)(\eta-1) r^{4}\right)+3 \pi^{3} C_{0}^{\prime} \sin \left(2 \pi\left(\alpha_{0}-\alpha_{1}\right)\right)\left(-49 \eta \zeta_{\perp}^{\prime 2}\right. \\
& \left.+13440 \pi(\eta-1) \zeta_{\perp}^{\prime} r^{3}+4032 \pi(\eta-1) \zeta_{\perp}^{\prime} r^{2}+147456 \pi^{2}(\eta-1) r^{4}-672 \pi \zeta_{\perp}^{\prime} r\right)+3 C_{1}(49(6 \eta \\
& \left.+\pi^{2}-3\right) \zeta_{\perp}^{\prime 2}-672 \pi \zeta_{\perp}^{\prime} r\left(2 \eta\left(\pi^{2}(r(20 r+9)+2)-3\right)-\pi^{2}(2 r(20 r+9)+5)+3\right) \\
& \left.\left.-221184 \pi^{4}(\eta-1) r^{4}\right)\right) \\
& \frac{\left\langle U_{y}\right\rangle}{L \omega_{0}} \approx-\frac{7 C_{0} C_{1} \zeta_{\perp}^{\prime}}{30 \pi^{3}\left(7 \eta \zeta_{\perp}^{\prime}+96 \pi r\right)\left(73728 \pi^{2} r^{4}+2688 \zeta_{\perp}^{\prime} \pi(r(5 r+3)+1) r+49 \zeta_{\perp}^{\prime 2}\right)^{2}}\left(45 C_{1}\right. \\
& \times\left(7 \zeta _ { \perp } ^ { \prime } \left(-4718592 \pi^{5}\left(4 \pi^{2}(5 r+2)-39\right) r^{6}+516096 \zeta_{\perp}^{\prime} \pi^{2}\left(\pi^{2}(r(35 r-6)+1)-6\right) r^{3}\right.\right. \\
& \left.+3136 \zeta_{\perp}^{\prime 2} \pi\left(4 \pi^{4}(5 r+2) r-72 r-3 \pi^{2}(r(90 r+29)+6)+36\right) r-1029 \zeta_{\perp}^{\prime 3}\left(-8+\pi^{2}\right)\right) \eta^{2} \\
& +\left(-1358954496 \pi^{6}\left(\pi^{2}(20 r+6)-15\right) r^{7}-16515072 \zeta_{\perp}^{\prime} \pi^{3}\left(-3 \pi^{2}\left(33 r^{2}+6 r+5\right)\right.\right. \\
& \left.+2 \pi^{4}(r(r(70 r+39)+12)+1)+18\right) r^{4}-301056 \zeta_{\perp}^{\prime 2} \pi^{2}\left(36(r-1)+3 \pi^{2}\left(r\left(80 r^{2}+74 r+39\right)\right.\right. \\
& \left.+10)+2 \pi^{4}(r(2 r(5 r(20 r+19)+46)+25)+4)\right) r^{2}-10976 \zeta_{\perp}^{\prime 3} \pi(-36(2 r+1) \\
& \left.\left.+2 \pi^{4}(4 r+1)(5 r+2)-3 \pi^{2}(4 r(25 r+6)+1)\right) r+2401 \zeta_{\perp}^{\prime 4}\left(-12+\pi^{2}\right)\right) \eta+32 \pi r\left(14155776 \pi^{5}\right. \\
& \times\left(\pi^{2}(60 r+22)-15\right) r^{6}+516096 \zeta_{\perp}^{\prime} \pi^{2}\left(-3 \pi^{2}(r(13 r+6)+3)+2 \pi^{4}(r(r(90 r+49)+9)+1)+9\right) \\
& \times r^{3}+9408 \zeta_{\perp}^{\prime 2} \pi\left(36 r+3 \pi^{2}\left(20 r^{3}+2 r^{2}+r+2\right)+2 \pi^{4}(r(2 r(5 r(20 r+17)+31)+15)+2)-18\right) r \\
& \left.\left.+343 \zeta_{\perp}^{\prime 3}\left(\pi^{4}(6 r+2)-3 \pi^{2}(4 r+1)(10 r+1)-36\right)\right)\right)+C_{0}^{\prime} \pi^{2}\left(3 \left(7 \zeta _ { \perp } ^ { \prime } \left(4 7 1 8 5 9 2 \pi ^ { 3 } \left(120 \pi^{2}(5 r+3)\right.\right.\right.\right. \\
& \left.+4 \pi^{4}(25 r+8)-585\right) r^{6}-172032 \zeta_{\perp}^{\prime} \pi^{2}\left(4 \pi^{4} r(10 r+1)+585(r(5 r+2)+1)-6 \pi^{2}(5 r(25 r(4 r+3)\right. \\
& +12)+11)) r^{3}-3136 \zeta_{\perp}^{\prime 2} \pi\left(4 \pi^{4} r(5 r+4)+\pi^{2}(72-84 r)-585(5 r(2 r+1)+2)\right) r+1029 \zeta_{\perp}^{\prime 3}(65 \\
& \left.\left.-6 \pi^{2}\right)\right) \eta^{2}+\left(452984832 \pi^{4}\left(30 \pi^{2}(25 r+13)+\pi^{4}(250 r+72)-585\right) r^{7}+16515072 \zeta_{\perp}^{\prime} \pi^{3}\right.
\end{aligned}
$$




$$
\begin{aligned}
& \times\left(-45(r(47 r+26)+13)+2 \pi^{4} r(r(175 r+59)+13)+6 \pi^{2}(5 r(r(115 r+72)+13)+11)\right) r^{4} \\
& +301056 \zeta_{\perp}^{\prime 2} \pi^{2}\left(-6 \pi^{2}(r(10 r+1)(5 r(10 r+9)-7)+12)+45(r(2 r(90 r+101)+101)+26)\right. \\
& \left.+2 \pi^{4}(r(r(10 r(50 r+49)+209)+62)+10)\right) r^{2}+10976 \zeta_{\perp}^{\prime 3} \pi(-45(90 r(2 r+1)+23) \\
& \left.\left.+2 \pi^{4}(r(10 r+33)+5)+12 \pi^{2}(r(25 r-12)+4)\right) r+2401 \zeta_{\perp}^{\prime 4}\left(-135+14 \pi^{2}\right)\right) \eta+32 \pi r \\
& \times\left(-14155776 \pi^{3}\left(30 \pi^{2}(25 r+9)+\pi^{4}(250 r+96)-405\right) r^{6}-516096 \zeta_{\perp}^{\prime} \pi^{2}(-405(r(5 r+2)+1)\right. \\
& \left.+2 \pi^{4} r(5 r(55 r+23)-1)+3 \pi^{2}(10 r(r(155 r+92)+19)+21)\right) r^{3}-9408 \zeta_{\perp}^{\prime 2} \pi(405(5 r(2 r+1)+2) \\
& \left.+2 \pi^{4} r(5 r(10 r(10 r+7)+7)+6)+6 \pi^{2}(r(5 r(60 r(5 r+4)+113)+147)+9)\right) r \\
& \left.\left.+343 \zeta_{\perp}^{\prime 3}\left(2 \pi^{2}\left(5 r\left(10\left(-3+\pi^{2}\right) r+\pi^{2}-12\right)+6\right)-405\right)\right)\right) \cos \left(2\left(\alpha_{0}-\alpha_{1}\right) \pi\right) \\
& +\pi\left(67947724800(\eta-1) \pi^{4}\left(-15+16 \pi^{2}\right) r^{8}+70778880 \pi^{3}\left(3 5 ( \eta - 1 ) \zeta _ { \perp } ^ { \prime } \left(2(7 \eta+19) \pi^{2}\right.\right.\right. \\
& \left.-15(2 \eta+5))+288 \pi\left(\eta\left(-39+17 \pi^{2}\right)-21 \pi^{2}+27\right)\right) r^{7}+5160960 \zeta_{\perp}^{\prime} \pi^{2}\left(144 \pi^{3}(\eta(18 \eta+43)\right. \\
& \left.-65)+175(\eta-1)(\eta+3) \zeta_{\perp}^{\prime} \pi^{2}-2625(\eta-1) \eta \zeta_{\perp}^{\prime}-432(\eta(26 \eta+97)-95) \pi\right) r^{6} \\
& +1548288 \zeta_{\perp}^{\prime} \pi^{2}\left(64 \pi^{3}(101 \eta-53)+175(3 \eta(\eta+2)-7) \zeta_{\perp}^{\prime} \pi^{2}+525 \eta(19-23 \eta) \zeta_{\perp}^{\prime}\right. \\
& +1920(28-31 \eta) \pi) r^{5}+258048 \zeta_{\perp}^{\prime} \pi^{2}\left(576(7 \eta-6) \pi\left(-15+\pi^{2}\right)+7 \zeta_{\perp}^{\prime}\left((\eta(224 \eta+333)-95) \pi^{2}\right.\right. \\
& -30(\eta(124 \eta-147)+5))) r^{4}+18816 \zeta_{\perp}^{\prime 2} \pi\left(-2160 \pi(\eta(28 \eta-41)+1)+144(\eta(28 \eta+83)-11) \pi^{3}\right. \\
& \left.+175\left(-4 \eta^{2}+\eta+3\right) \zeta_{\perp}^{\prime} \pi^{2}+1050 \eta(7 \eta-1) \zeta_{\perp}^{\prime}\right) r^{3}+28224 \zeta_{\perp}^{\prime 2} \pi\left(960 \pi(\eta+2)+64(29 \eta-2) \pi^{3}\right. \\
& \left.+7(\eta(34-33 \eta)+5) \zeta_{\perp}^{\prime} \pi^{2}+105 \eta(17 \eta-1) \zeta_{\perp}^{\prime}\right) r^{2}-32928 \zeta_{\perp}^{\prime 3} \pi\left((\eta(4 \eta-27)+5) \pi^{2}-15(\eta(4 \eta+3)\right. \\
& \left.\left.\left.+5)) r+12005(\eta-1) \eta \zeta_{\perp}^{\prime 4}\left(-15+\pi^{2}\right)\right) \sin \left(2\left(\alpha_{0}-\alpha_{1}\right) \pi\right)\right)\right) .
\end{aligned}
$$

Here $r=R / L$ is the dimensionless radius of the bead and $\zeta_{\perp}^{\prime}=\zeta_{\perp} / \mu=2 \zeta_{\|} / \mu \sim$ $8 \pi /(\log (2 L / a)+0.5) \sim 4.1$ where $a \sim 100 \mathrm{~nm}$ is the radius of axoneme, $L \sim 10 \mu \mathrm{m}$ is the contour length of axoneme and we have assumed $\eta=\zeta_{\perp} / \zeta_{\|}=2$. Note that in the absence of the bead $(r=0)$ and with $\eta=0.5$, Eqs. S.16-S.18 simplify to:

$$
\begin{aligned}
& \frac{\left\langle\Omega_{z}\right\rangle}{\omega_{0}} \approx-0.42 C_{0} C_{1}^{2}\left(1-1.46 \frac{C_{0}^{\prime}}{C_{1}} \cos (\gamma)\right), \\
& \frac{\left\langle U_{x}\right\rangle}{L \omega_{0}} \approx+0.16 C_{1}^{2}\left(-1+1.14 \frac{C_{0}^{\prime}}{C_{1}} \cos (\gamma)-1.57 \frac{C_{0}^{\prime}}{C_{1}} \sin (\gamma)\right), \\
& \frac{\left\langle U_{y}\right\rangle}{L \omega_{0}} \approx+0.038 C_{0} C_{1}^{2}\left(1-1.58 \frac{C_{0}^{\prime}}{C_{1}} \cos (\gamma)-1.79 \frac{C_{0}^{\prime}}{C_{1}} \sin (\gamma)\right) .
\end{aligned}
$$

which are previously discussed in Ref. [26].

Furthermore, in the limit of small $1 / r$ (bead size $R$ much larger than the length of 
flagella $L$ ), we obtain up to the second order:

$$
\begin{aligned}
\frac{\left\langle\Omega_{z}\right\rangle}{\omega_{0}} \approx & \frac{7 C_{0} C_{1}(\eta-1) \zeta_{\perp}^{\prime} r^{-2}}{384 \pi}\left(18 \pi C_{1}+\left(15-16 \pi^{2}\right) C_{0}^{\prime} \sin \left(2 \pi\left(\alpha_{0}-\alpha_{1}\right)\right)-5 \pi\left(3+\pi^{2}\right) C_{0}^{\prime} \cos \left(2 \pi\left(\alpha_{0}-\alpha_{1}\right)\right)\right) \\
\frac{\left\langle U_{x}\right\rangle}{L \omega_{0}} \approx & \frac{7 C_{1}(\eta-1) \zeta_{\perp}^{\prime} r^{-1}}{110592 \pi^{2}}\left(3 \pi C_{0}^{\prime} \sin \left(2 \pi\left(\alpha_{0}-\alpha_{1}\right)\right)\left(7(4 \eta+5) \zeta_{\perp}^{\prime} r^{-1}-384 \pi\right)+C_{0}^{\prime} \cos \left(2 \pi\left(\alpha_{0}-\alpha_{1}\right)\right)\right. \\
& \left.\times\left(7\left(4\left(3+\pi^{2}\right) \eta+5 \pi^{2}\right) \zeta_{\perp}^{\prime} r^{-1}-384 \pi\left(3+\pi^{2}\right)\right)+3 C_{1}\left(576 \pi-7(6 \eta+5) \zeta_{\perp}^{\prime} r^{-1}\right)\right) \\
\frac{\left\langle U_{y}\right\rangle}{L \omega_{0}} & \approx-\frac{7 C_{0} C_{1} \zeta_{\perp}^{\prime} r^{-1}}{1105920 \pi^{2}}\left(5 C _ { 0 } ^ { \prime } \operatorname { s i n } ( 2 \pi ( \alpha _ { 0 } - \alpha _ { 1 } ) ) \left(35(\eta-1)\left(75-2 \pi^{2}(9 \eta+61)\right) \zeta_{\perp}^{\prime} r^{-1}\right.\right. \\
& \left.+288 \pi\left(\left(17 \pi^{2}-39\right) \eta-21 \pi^{2}+27\right) r^{-1}+960 \pi\left(16 \pi^{2}-15\right)(\eta-1)\right)+3 C_{0}^{\prime} \cos \left(2 \pi\left(\alpha_{0}-\alpha_{1}\right)\right) \\
& \times\left(8000 \pi^{2}\left(3+\pi^{2}\right)(\eta-1)-r^{-1}\left(175 \pi^{3}(\eta-1)(2 \eta+13) \zeta_{\perp}^{\prime}+175 \pi(\eta-1)(2 \eta+19) \zeta_{\perp}^{\prime}\right.\right. \\
& \left.\left.-768 \pi^{4}(3 \eta-4)-960 \pi^{2}(13 \eta-9)+1440(13 \eta-9)\right)\right)+30 C_{1}\left(35 \pi(\eta-1)(4 \eta+21) \zeta_{\perp}^{\prime} r^{-1}\right. \\
& \left.\left.+720(3 \eta-1) r^{-1}-96 \pi^{2}\left(9 \eta r^{-1}+30 \eta-11 r^{-1}-30\right)\right)\right) .
\end{aligned}
$$

In the opposite limit of small $r$ (i.e. $R \ll L$ ), up to the leading order, we set $\eta=0.5$ and $\zeta_{\perp}^{\prime}=4.33$ to get:

$$
\begin{aligned}
\frac{\left\langle\Omega_{z}\right\rangle}{\omega_{0}} \approx-1368.76 C_{0} C_{1}\left(C_{0}^{\prime} r(0.0186479-1.56589 r) \sin \left(2 \pi\left(\alpha_{0}-\alpha_{1}\right)\right)+C_{0}^{\prime}(r(1.09939 r+0.0164496)\right. \\
\left.\quad-0.00284628) \cos \left(2 \pi\left(\alpha_{0}-\alpha_{1}\right)\right)+C_{1}(r(r-0.0372754)+0.00194639)\right), \\
\frac{\left\langle U_{x}\right\rangle}{L \omega_{0}} \approx-960.229 C_{1}\left(C_{0}^{\prime}(r(0.0651089-2.49374 r)-0.00163586) \sin \left(2 \pi\left(\alpha_{0}-\alpha_{1}\right)\right)+C_{0}^{\prime}(r(0.0267323\right. \\
\left.\quad-0.704702 r)-0.00119235) \cos \left(2 \pi\left(\alpha_{0}-\alpha_{1}\right)\right)+C_{1}(r(r-0.0310872)+0.00104142)\right),
\end{aligned}
$$

$$
\begin{aligned}
& \frac{\left\langle U_{y}\right\rangle}{L \omega_{0}} \approx-941 . C_{0} C_{1}\left(C_{0}^{\prime}(r(0.0862795-4.80748 r)+0.000454339) \sin \left(2 \pi\left(\alpha_{0}-\alpha_{1}\right)\right)+C_{0}^{\prime}(r(-1.05\right. \\
&\left.-0.00632217)+0.00040078) \cos \left(2 \pi\left(\alpha_{0}-\alpha_{1}\right)\right)+C_{1}(r(r-0.0100976)-0.000253701)\right) .
\end{aligned}
$$

\subsection{Rotational velocity of a bead attached asymmetrically to a freely-swimming axoneme}

To mimic a sperm-like swimmer, we consider a model flagellum with only the main traveling wave component $C_{1}$, setting the other modes to zero $\left(C_{0}^{\prime}=C_{1}^{\prime}=C_{2}=0\right)$. We calculate the mean rotational velocity of an axoneme attached sideways to a bead using 
the matrix introduced in Eq. S.15 and drag matrix of flagellum to obtain:

$$
\begin{aligned}
\left\langle\Omega_{z}\right\rangle / \omega_{0} & \approx-\left(8 0 6 4 C _ { 1 } ^ { 2 } \zeta _ { \perp } ^ { \prime } r y _ { b } \left(7 \eta ^ { 2 } \zeta _ { \perp } ^ { \prime } \left(343 \zeta_{\perp}^{\prime 3}\left(6+\pi^{2}\right)-2352 \zeta_{\perp}^{\prime 2} \pi r\left(-24+\pi^{2}\right.\right.\right.\right. \\
& \left.\times\left(-3+40 r^{2}-12 x_{b}\right)\right)-5308416 \pi^{5} r^{3}\left(5 r^{2}-3 x_{b}^{2}\right)-129024 \zeta_{\perp}^{\prime} \pi^{2} r^{2} \\
& \left.\times\left(-3+\pi^{2}\left(-1+25 r^{2}-12 x_{b}^{2}\right)\right)\right)+288 \pi r\left(-343 \zeta_{\perp}^{\prime 3}+21504 \zeta_{\perp}^{\prime} \pi^{2} r^{2}\right. \\
& \times\left(-3+\pi^{2}\left(-1+50 r^{2}-24 x_{b}^{2}-30 y_{b}^{2}\right)\right)+784 \zeta_{\perp}^{\prime 2} \pi r\left(-12+\pi^{2}\left(1+40 r^{2}\right.\right. \\
& \left.\left.\left.-12 x_{b}-24 y_{b}^{2}\right)\right)+3538944 \pi^{5} r^{5}\right)+3 \eta\left(-2401 \zeta_{\perp}^{\prime 4}+5488 \zeta_{\perp}^{\prime 3} \pi^{3} r\right. \\
& \times\left(3+40 r^{2}-12 x_{b}\right)-4128768 \zeta_{\perp}^{\prime} \pi^{3} r^{3}\left(-3+\pi^{2}\left(-1+10 r^{2}-3 x_{b}^{2}-15 y_{b}^{2}\right)\right) \\
& -339738624 \pi^{6} r^{6}+75264 \zeta_{\perp}^{\prime 2} \pi^{2} r^{2} \times\left(18+\pi^{2}\left(1+60 r^{2}+12\left(1-4 x_{b}\right) x_{b}\right.\right. \\
& \left.\left.\left.\left.\left.+24 y_{b}^{2}\right)\right)\right)\right)\right) /\left(2 \pi ^ { 2 } \left(( 7 \eta \zeta _ { \perp } ^ { \prime } + 9 6 \pi r ) \left(49 \zeta_{\perp}^{\prime 2}+2688 \zeta_{\perp}^{\prime} \pi r\left(1+5 r^{2}-3 x_{b}\right)\right.\right.\right. \\
& \left.\left.\left.+36864 \pi^{2} r^{2}\left(5 r^{2}-3 x_{b}^{2}\right)\right)-110592 \pi^{2} r^{2}\left(7 \zeta_{\perp}^{\prime}+96 \pi r\right) y_{b}^{2}\right)^{2}\right)
\end{aligned}
$$

where $x_{b}^{2}+y_{b}^{2}=r^{2}, \zeta_{\perp}^{\prime}=\zeta_{\perp} / \mu=4.33$ and $\eta=\zeta_{\|} / \zeta_{\perp}=0.5$.

\subsection{Supplementary figures}
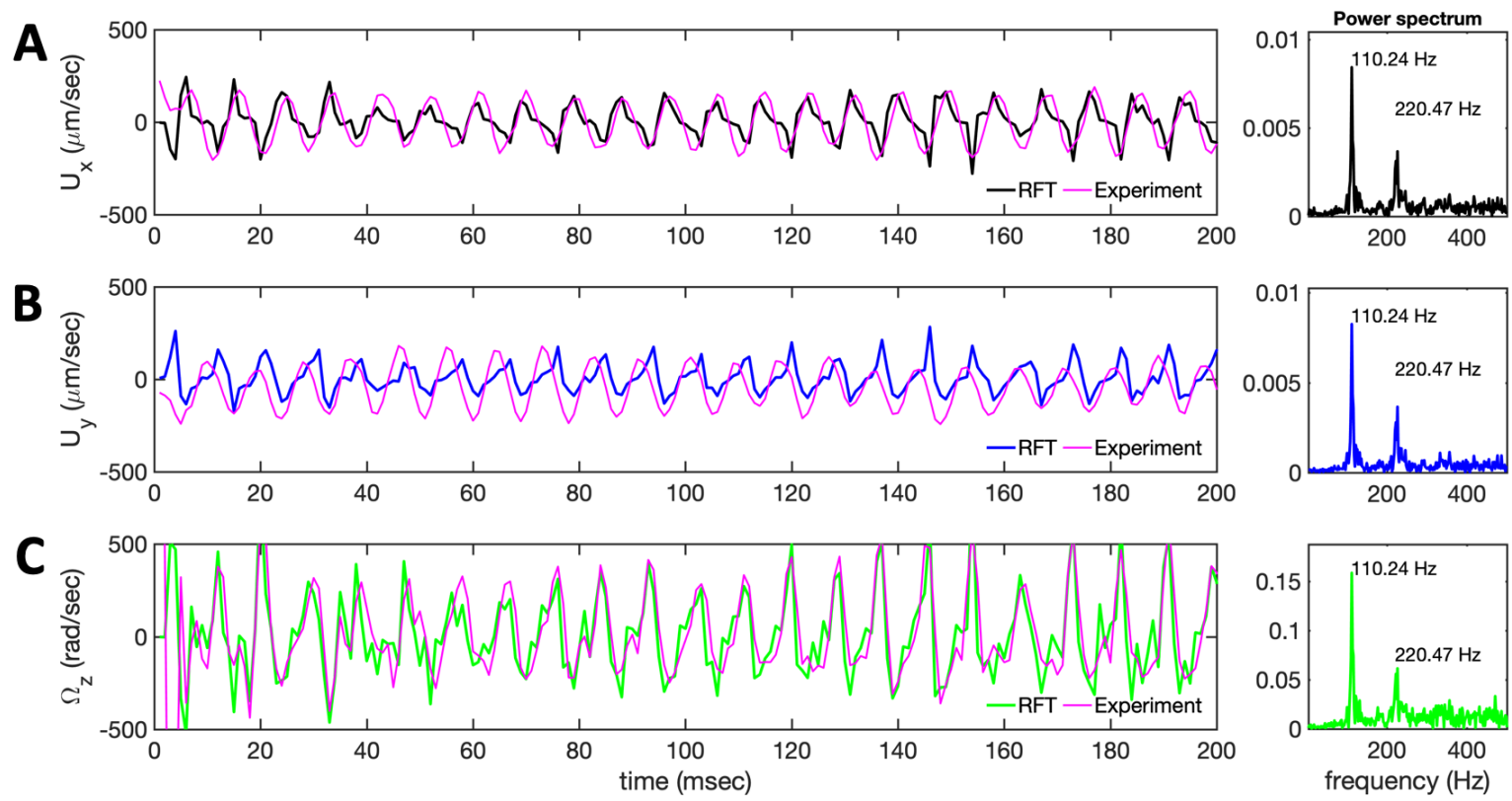

Figure S2. A-C) Velocity components of the bead's center $U_{x}(t)$ and $U_{y}(t)$, and the rotational velocity of the bead $\Omega_{z}(t)$ measured in the body-fixed frame of the exemplary axoneme in Video 2. Comparison with results obtained in the frame work of resistiveforce theory shows a good agreement. The power spectra show a dominant peak at the beat frequency of $110.24 \mathrm{~Hz}$ and its second harmonic. 

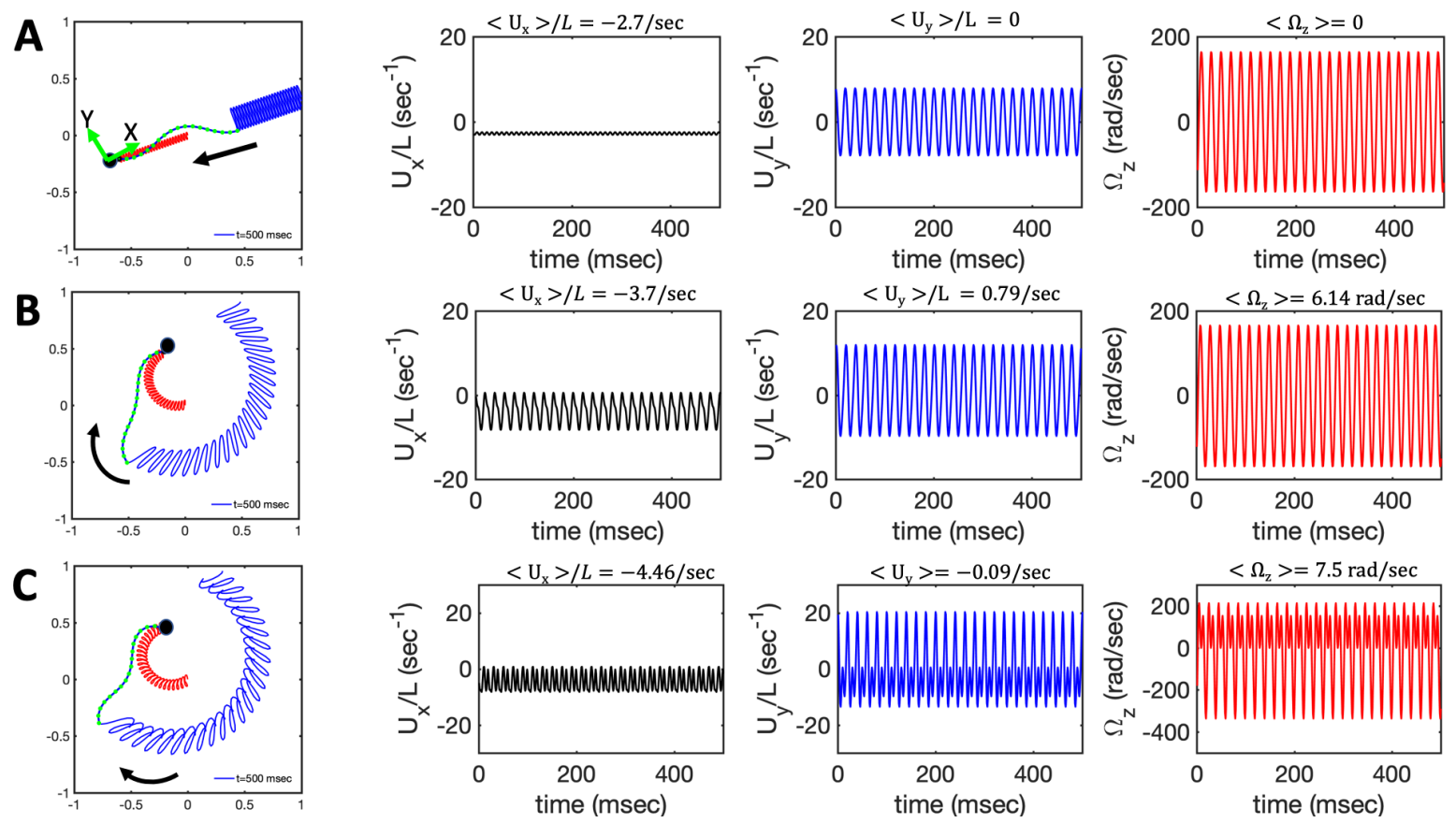

Figure S3. Simulations to show the effect of $C_{0}$ and $C_{2}$ at fixed amplitude of dynamic mode $C_{1}=0.5$. A bead of radius $R / L=0.1$ is attached to the basal end. A-C) Swimming trajectory and mean translational and rotational velocities of the swimmer in the body-fixed frame. Parameters are $f_{0}=50 \mathrm{~Hz}, \eta=\zeta_{\perp} / \zeta_{\|}=1.8$, A) $C_{0}=C_{2}=0$, B) $C_{0}=0.25, C_{2}=0$, and C) $C_{0}=C_{2}=0.25$.

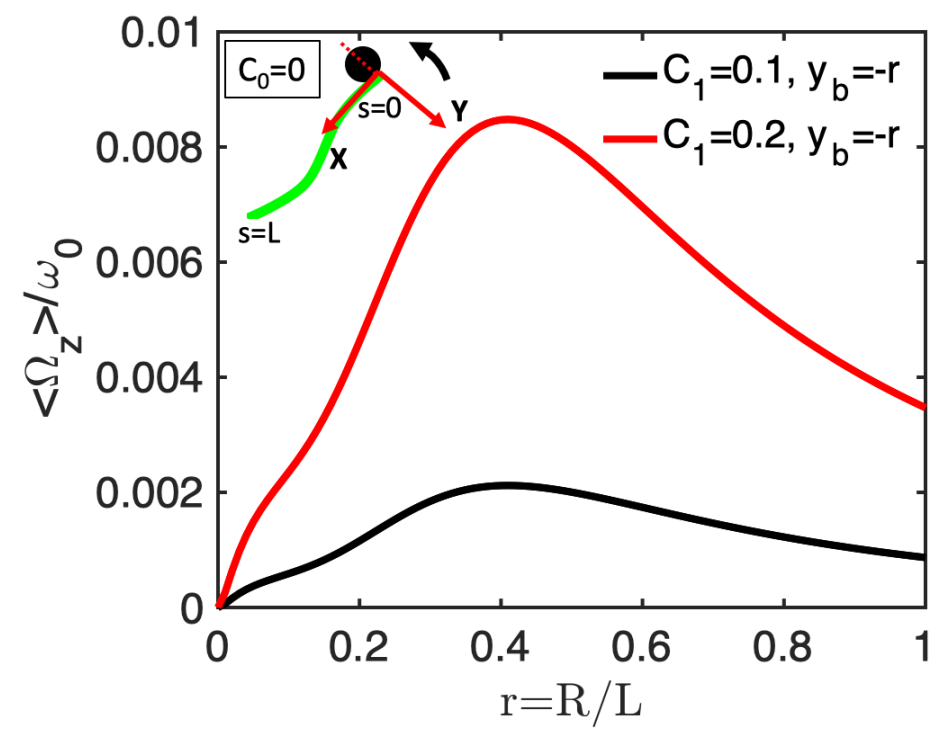

Figure S4. Mean rotational velocity of a bead which is asymmetrically attached to an axoneme with $y_{b}=-r$ and $x_{b}=0$ for two different values of $C_{1}$. 

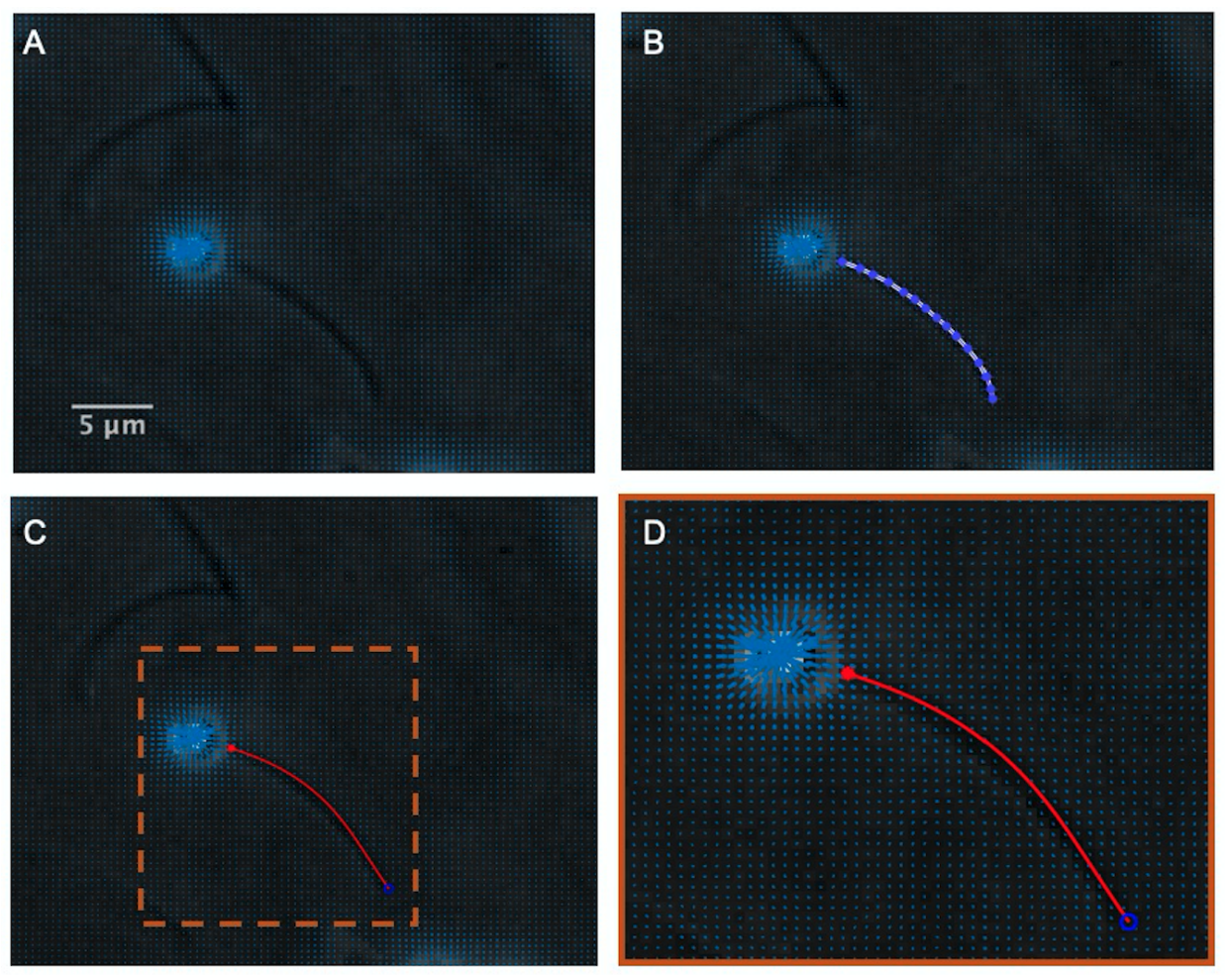

Figure S5. Gradient vector flow method implemented to track the flagellum. High intensity of the bead interferes with tracking, therefore it is removed by thresholding method. 